


\section{Manuel pratique de production durable des gommes au Burkina Faso}

Damas Poda, Mathurin Zida, Serges Zoubga, Honoré Béréoudougou, Attina Lankoandé, Jean Emmanuel Zoungrana, Daniel Tiveau 


\section{Remerciements}

L' élaboration de ce guide a été financée par la FAO et l'Asdi et sa publication par l'Asdi. Les auteurs expriment leurs remerciements à ces organisations.

Le contenu du document ne représente pas nécessairement les points de vue des institutions pour lesquelles travaillent les auteurs ni des partenaires financiers qui ont sponsorisé le document.

\section{Collaborateurs}

Direction des Forêts, DGCN, MECV, Burkina Faso : Damas Poda, Serges Zoubga, Honoré Béréoudougou, Attina Lankoandé, Jean Emmanuel Zoungrana

CIFOR : Mathurin Zida, Daniel Tiveau

Sources des photos : CIFOR, DiFor, DTA

(C) par CIFOR

Tous droits réservés.

Publié en 2009

Direction des Forêts - Direction Générale de la Conservation de la Nature Ministère de l'Environnement et du Cadre de Vie 01 BP 6429 Ouagadougou 01, Burkina Faso Tél. : +22650316119

CIFOR - Bureau régional Afrique Occidentale 06 BP 9478 Ouagadougou 06, Burkina Faso

Tél. : +22650304742 Fax : +22650302930

Site web : www.cifor.cgiar.org E-mail : cifor-westafrica@cgiar.org 


\section{Table des matières}

Sigles et abréviations

Liste des photographies

vi

Liste des tableaux

vi

Liste des figures

vi

Avant-propos

vii

Introduction

Module I. Généralités

4

\subsection{Définitions}

4

1.1.1. Les gommes

1.1.2. Les gommiers 5

1.1.3. La gommeraie 10

1.1.4. Classification des gommes 10

1.1.5. Les résines 
1.2. Utilité des espèces gommifères et de la gomme

1.2.1. Utilité des acacias gommiers 12

1.2.2. Utilité de la gomme 14

Module II. Aménagement et gestion d'une gommeraie $\quad 16$

2. 1. La méthode naturelle 16

2.1.1. Jeunes plants de gommiers épargnés volontairement dans le champ lors des labours et entretenus $\quad 16$

2.1.2. Mise en défens 17

2.1.3. Pacage et mise en stabulation des animaux dans une aire définie $\quad 17$

2.2. Méthode artificielle de réalisation de gommeraie 18

2.2.1. Plantation de gommeraie 18

2.2.2. Le semi direct 26

2.3. L'entretien de la gommeraie 26

2.4. Gestion de la gommeraie 30

Module III. Techniques de production de la gomme 32

3.1. La gommose 32

3.2. Les techniques de production 32

3.2.1. Matériel de saignée et de récolte 32

3.2.2. La méthode traditionnelle de saignée 34

3.2.3. La technique moderne de saignée 34

3.3. Récolte de la gomme 37

3.4. Le traitement et le conditionnement de la gomme 39

3.5. Valorisation de la gomme $\quad 40$

3.5.1. A l'échelle internationale $\quad 41$

3.5.2. Le marché de la gomme au Burkina Faso 42

3.5.3. Les applications de la gomme au Burkina Faso 43

Documents consultés $\quad 44$ 


\section{Sigles et abréviations}

$\mathrm{ABN}$

AIDGUM

APEGA

Asdi

CIFOR

CNSF

DGCN

DiFor

DRS/CES

DTA

FAO

JECFA

MECV

NGARA

PFNL

PLCE/BN

RNA
Autorité du Bassin du fleuve Niger

Association Internationale pour le Développement des Gommes Naturelles

Association des Professionnels et Exportateurs de Gomme Arabique

Agence Suédoise de coopération pour le Développement International

Center for International Forestry Research

Centre National de Semences Forestières

Direction Générale de la Conservation de la Nature

Direction des Forêts

Défense et Restauration des Sols/Conservation des Eaux et des Sols

Département des Technologies Alimentaires (Institut de Recherche en Sciences Appliquées et Technologies)

Organisation des Nations Unies pour l'Alimentation et l'Agriculture

Joint Expert Committee on Food Additives

Ministère de l'Environnement et du Cadre de Vie

Network for Natural Gums and Resins in Africa

Produits Forestiers Non Ligneux

Programme de Lutte Contre l'Ensablement du Bassin du fleuve Niger

Régénération Naturelle Assistée 


\section{Liste des photographies}

Photo 1. Nodule de gomme 4

Photo 2. Acacia laeta 6

$\begin{array}{lll}\text { Photo 3. Acacia senegal } & 7\end{array}$

Photo 4. Acacia seyal 8

Photo 5. Acacia dudgeoni $\quad 9$

Photo 6. Gomme d'Acacia senegal 11

Photo 7. Nodules de gomme d'Acacia prêtes pour la vente $\quad 11$

$\begin{array}{lll}\text { Photo 8. } & \text { Champ de culture sous peuplement d'Acacia }\end{array}$

Photo 9. Reconstitution du couvert végétal à partir de plantations d'Acacia senegal 13

$\begin{array}{lll}\text { Photo 10. Peuplement naturel d'Acacia à Bogandé } & 18\end{array}$

Photo 11. Une gommeraie artificielle d'Acacia senegal à Mani 19

Photo 12. Sous-solage à la charrue Delfino d'une parcelle de plantation à Tougouri 23

Photo 13. Outils couramment utilisés au Burkina Faso pour la saignée et la récolte de la gomme

Photo 14. Indication de la grosseur requise pour une branche à saigner

Photos 15 à 20. Les différentes étapes dans la réalisation de la saignée 35-36

Photo 21. Care exsudant de la gomme 38

\section{Liste des tableaux}

$\begin{array}{ll}\text { Tableau 1. Différents usages des gommes } & 15\end{array}$

Tableau 2. Cycle d'exploitation d'une gommeraie 30

\section{Liste des figures}

Figure 1. Carte de l'aire de distribution d'Acacia senegal et d'Acacia laeta au Burkina Faso

Figure 2. Opérations de mise en terre d'un plant en pot 28

Figure 3. Cycle de gestion d'une gommeraie 31

Figure 4. Opération de récolte de la gomme 38 


\section{Avant-propos}

T es gommes en général et la gomme arabique en particulier sont des produits de base qui font l'objet aujourd'hui d'un commerce international florissant et le développement de la filière des gommes au Burkina Faso pourrait contribuer à lutter contre la pauvreté en milieu rural. C'est dans cette perspective que plusieurs initiatives ont été lancées depuis la fin des années 70. La volonté des pouvoirs publics de promouvoir les gommiers et la gomme arabique sest notamment traduite par lélaboration de documents de politique et de stratégie ad hoc ainsi que la mise en œuvre de divers projets associés.

Le projet «GTF/RAF 387/ITA - Opération Acacia » en est un exemple. Financé par la coopération italienne, ce projet a contribué au renforcement des capacités des agents forestiers et des producteurs en matière de production de gomme et de gestion des peuplements gommiers. C'est pour pallier à l'insuffisance de supports de vulgarisation des connaissances techniques sur ces thèmes que le projet a entrepris de capitaliser sous la forme d'un guide pratique les sessions de formation qu'il a eu à dispenser.

Linitiative CIFOR-ASDI sur les Produits Forestiers Non Ligneux (PFNL) est un autre exemple de projet $^{1}$, représentatif cette fois de lintérêt des partenaires internationaux pour la gomme arabique en tant que PFNL pouvant contribuer à la réalisation des Objectifs de Développement du Millénaire dans les zones sèches d'Afrique. Dans cette perspective, ce projet a ciblé, entre autres, les actions de renforcement des capacités techniques des acteurs à la base en matière de production et de commercialisation des gommes au Burkina Faso. Cela est facilité par la mise à leur disposition de supports didactiques appropriés.

C'est en raison de cette convergence d'intérêts que la Direction des Forêts et le CIFOR se sont associés, à travers les deux initiatives susmentionnées,

\footnotetext{
${ }^{1}$ Le titre du projet est : "Atteindre les Objectifs du Millénaire pour le Développement dans les forêts sèches d'Afrique : de l'action locale aux réformes de la politique forestière nationale"; il est couramment appelé «Projet Forêt Sèche - 2e phase ».
} 
pour élaborer le présent " Manuel pratique de production durable des gommes au Burkina Faso » qui sera aussi adapté en langues nationales (mooré, gulmanchéma et fulfuldé).

Ce manuel est un outil de vulgarisation destiné aux techniciens de terrain et aux producteurs menant des activités de production de gomme et de gestion des peuplements gommiers. Il peut servir également aux formateurs et constitue de ce fait une source d'inspiration pour déventuels documents tels que des guides de formation pour d'autres utilisateurs de la filière. Adapté au contexte du Burkina Faso, il s'inspire de deux manuels de base élaborés par la FAO, NGARA et AIDGUM à savoir " Gommiers et gomme arabique, manuel de terrain » et «Programme de formation régionale».

Ce manuel n'a pas la prétention de traiter de façon exhaustive tous les domaines liés à la production et à la commercialisation de la gomme.

Le regroupement thématique a été fait délibérément dans le souci de diffuser au public cible les connaissances pratiques de base utiles à leurs activités. Les techniques de production de plants, de plantation, daménagement ont déjà été suffisamment décrites dans d’autres manuels de vulgarisation existants tels que le « Manuel de foresterie villageoise » et il ne nous a pas paru opportun de nous y attarder.

Toutes les suggestions à la Direction des Forêts ${ }^{2}$ après usage de ce document seront les bienvenues et serviront pour une relecture future du document.

\footnotetext{
2 Direction des Forêts - Direction Générale de la Conservation de la Nature - Ministère de l'Environnement et du Cadre de Vie 01 BP 6429 Ouagadougou 01, Burkina Faso.
} 


\section{Introduction}

T a gomme est un produit aussi vieux que le monde. Dans l'Egypte ancienne, elle était utilisée pour la fabrication de lencre, des hiéroglyphes, dans la poterie. De nos jours, ses propriétés physicochimiques compatibles avec de multiples utilisations dans les industries agroalimentaires, pharmaceutiques, cosmétiques et autres en font un produit de grand commerce au niveau international. Bien que la demande mondiale de gomme ait enregistré des fluctuations importantes au cours du $20^{\circ}$ siècle, avec des extrêmes à 60000 tonnes à la fin des années 60 et environ 20000 tonnes au début des années 90, les tendances sont maintenant à la hausse et les seules exportations des pays africains dépassaient les 40000 tonnes vers la fin des années 2000 (Fagg et Allison, 2004).

Le Soudan se taille la part du lion parmi les pays exportateurs de gomme arabique, suivi par le Tchad et le Nigeria. Quelques autres pays producteurs émergent en Afrique de l'Ouest avec le Sénégal et le Mali en tête, suivis de loin par le Niger et la Mauritanie.

Nonobstant une superficie en peuplements naturels d'acacias gommiers estimée à plus de 286000 ha et une production brute potentielle annuelle estimée entre 1500 et 4500 tonnes (Nikiéma et al., 1997), le Burkina Faso demeure quasiment méconnu sur la scène commerciale internationale de la gomme, ses exportations étant minimes (environ 80 tonnes $^{3}$ en 2005, selon les statistiques officielles de la Douane, à destination de la France, du Niger et du Nigeria).

Un diagnostic récent de la filière des gommes au Burkina Faso (Direction des Forêts, 2006) a identifié de nombreux facteurs qui constituent des contraintes pour le développement du potentiel dont dispose le pays. Ce sont, entre autres :

- la faiblesse des capacités techniques, organisationnelles et de gestion des producteurs ;

\footnotetext{
${ }^{3}$ Ce chiffre devrait être majoré pour tenir compte des exportations via les circuits informels, à destination du Niger principalement.
} 
- la faible productivité des peuplements d’acacias gommiers;

- l'irrégularité et la faiblesse des quantités de gomme collectée et l'inconstance de sa qualité ;

- la disparition des peuplements dans certaines zones due aux aléas climatiques et à l'effet de l'homme ;

- la faiblesse du prix d'achat aux producteurs et le statut foncier des peuplements.

Malgré ces contraintes, les activités de la filière connaissent depuis quelques temps une évolution positive favorisée par un certain nombre d'atouts qui peuvent se résumer comme suit :

- un potentiel important de peuplements naturels;

- un marché international porteur caractérisé par une tendance à la hausse de la demande ;

- la possibilité d'accroissement des rendements ;

- une prise de conscience des acteurs;

- l'existence d'un intérêt de plus en plus accru des bailleurs et des institutions de financement.

Cette situation montre l'ampleur des efforts à fournir pour permettre au Burkina de tirer pleinement partie de l'opportunité quoffre le commerce international des gommes pour générer des devises pour le pays et des revenus pour les petits producteurs ruraux.

Le renforcement des capacités techniques des producteurs peut contribuer significativement à résoudre certains des problèmes rencontrés par la filière, et la production du présent manuel participe à cet effort.

Le thème de «Production durable de la gomme " que le manuel se propose de traiter de manière pratique, veut concilier d'une part la nécessité d'une gestion durable de la ressource, pour assurer la pérennité du produit et capter tous les bénéfices environnementaux procurés par les gommiers, et d'autre part, la nécessité d'une production soutenue et de qualité de la gomme pour répondre aux exigences du marché et générer des revenus substantiels pour les producteurs.

La notion de production durable de la gomme a donc des implications, en termes de maitrise technique, à différents niveaux :

- en amont de la production, un aménagement et une gestion des gommeraies conformément aux principes de gestion durable ;

- au niveau de la production proprement dite, le respect d'un itinéraire technique approprié à même d’assurer une productivité élevée de l’arbre 
et une haute qualité de la gomme sans que les techniques utilisées soient dommageables à l'arbre ;

- au niveau de la post production immédiate, la mise en œuvre de techniques éprouvées à même de maintenir voire d’améliorer la qualité de la gomme récoltée, afin d'assurer la fourniture d'une gomme de haute valeur commerciale à l'industrie de transformation.

Le manuel est structuré de manière à mettre en exergue les éléments techniques dont la maîtrise permettra aux producteurs de s'inscrire dans une dynamique de production durable de la gomme. Il comporte trois (3) modules

- le premier fournit des informations générales sur les acacias gommiers, les gommes, les résines et leurs utilisations ;

- le second, traite des techniques d'aménagement et de gestion d'une gommeraie ;

- enfinle dernier détailleles techniques de production et depost production immédiate et aborde la question de la liaison avec le marché. 


\section{Module I Généralités}

\subsection{Définitions}

\subsubsection{Les gommes}

Sous l'effet d'une blessure de l'arbre par un agent quelconque (le vent, les animaux, l'homme, les insectes) ou d'un stress, certains arbres laissent couler un liquide sous forme de larme ou de boules qui durcit par la suite au contact de l'air. Cet exsudat est appelé gomme.

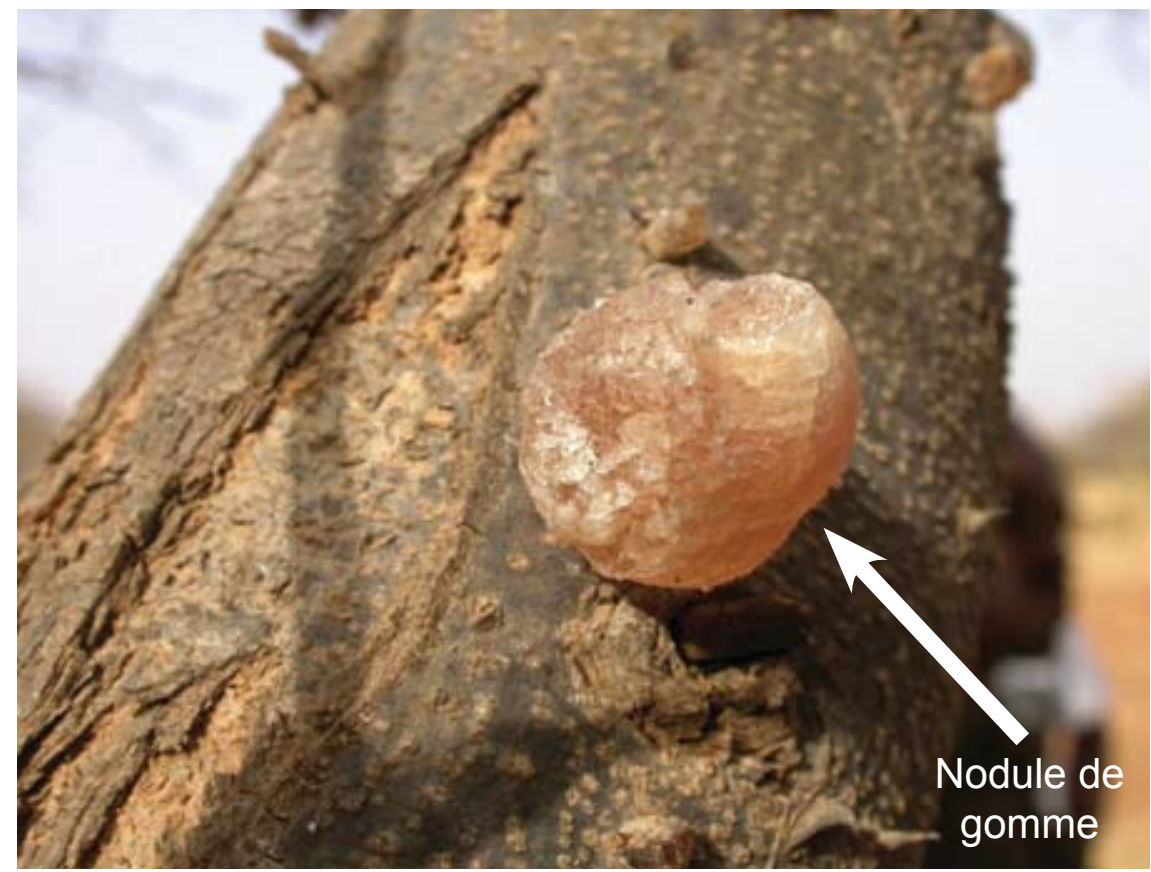

Photo 1. Nodule de gomme (CIFOR). 


\subsubsection{Les gommiers}

Les principales espèces productrices de la gomme, rencontrées au Burkina Faso, appartiennent aux genres Acacia, Combretum et Sterculia. Les gommes comestibles ou rencontrées sur les marchés proviennent de l'exsudation des espèces suivantes :

- Pour le genre Acacia :

Acacia senegal

Acacia laeta

Acacia seyal

Acacia gourmaensis

Acacia dudgeoni

Acacia raddiana

- Pour le genre Combretum :

Combretum aculeatum

Combretum nigricans

Combretum molle

- Pour le genre Sterculia :

Sterculia setigera

Les principaux acacia gommiers rencontrés au Burkina Faso (Acacia senegal, Acacia laeta, Acacia dudgeoni et Acacia seyal) se différencient essentiellement par la taille de leurs feuilles, la forme et la position des épines, l'aspect des fleurs, la forme des gousses. Certains se partagent les mêmes aires écologiques tandis que d'autres se rencontrent dans des isohyètes bien définies.

Du fait de la similitude des caractères morphologiques, il existe souvent une confusion botanique entre certains Acacia gommiers en loccurrence Acacia senegal, Acacia laeta et Acacia dudgeoni.

Von Maydel (1983) a fait une description de ces espèces avec des photos pour les illustrer.

Les photos ci-après présentent les caractéristiques de quelques Acacia gommiers. 

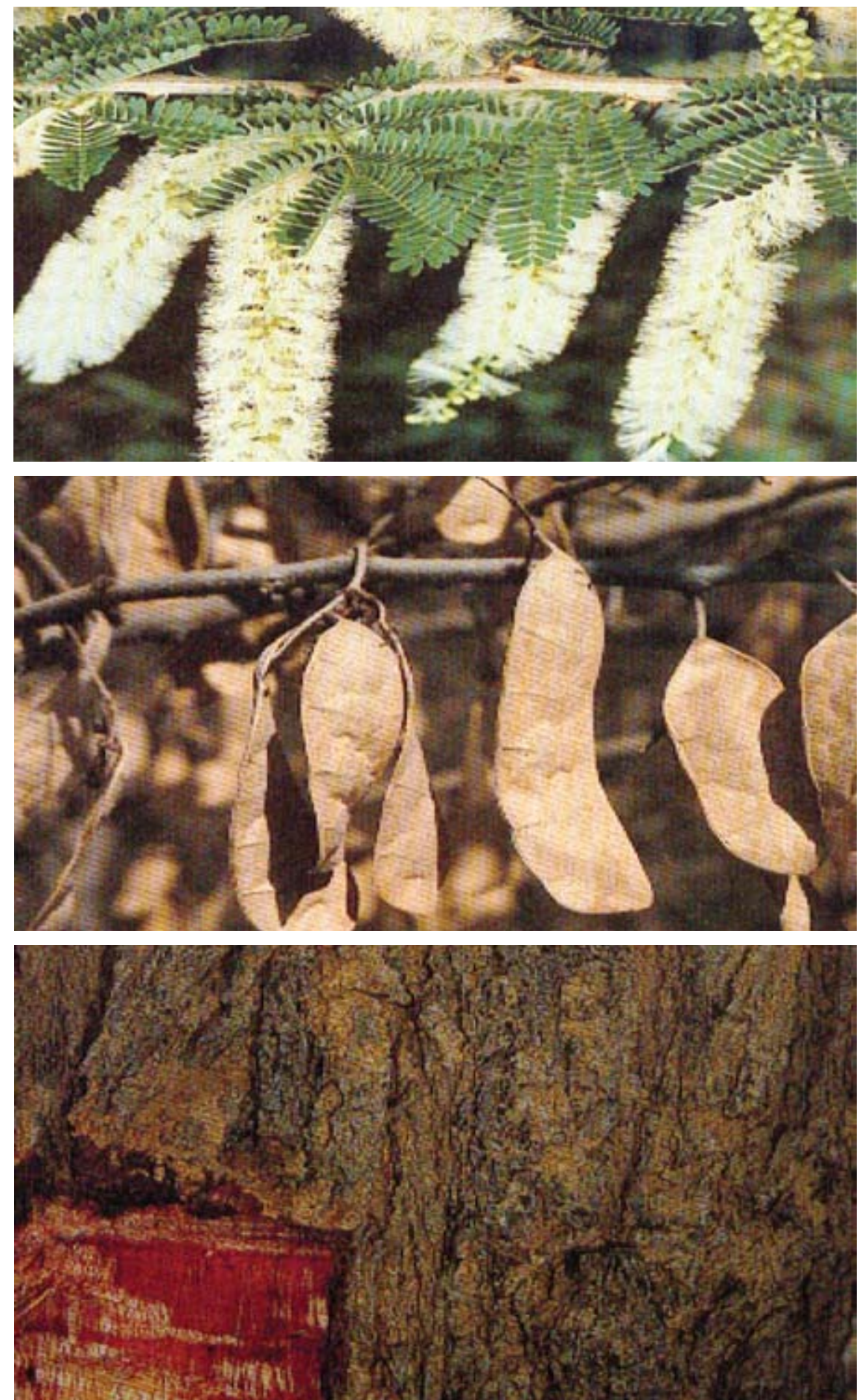

Photo 2. Acacia laeta (C DTA). 

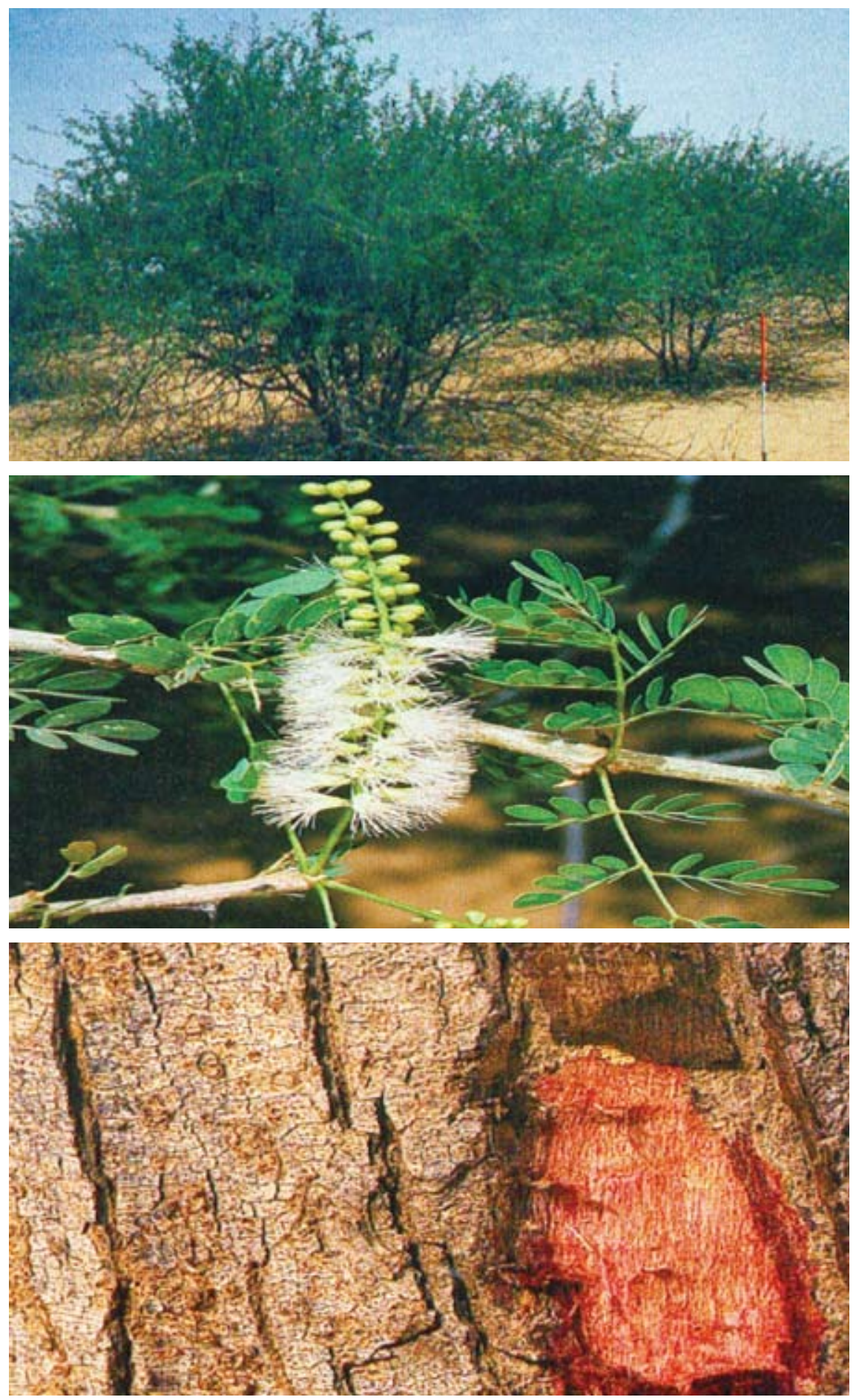

Photo 3. Acacia senegal (C DTA). 

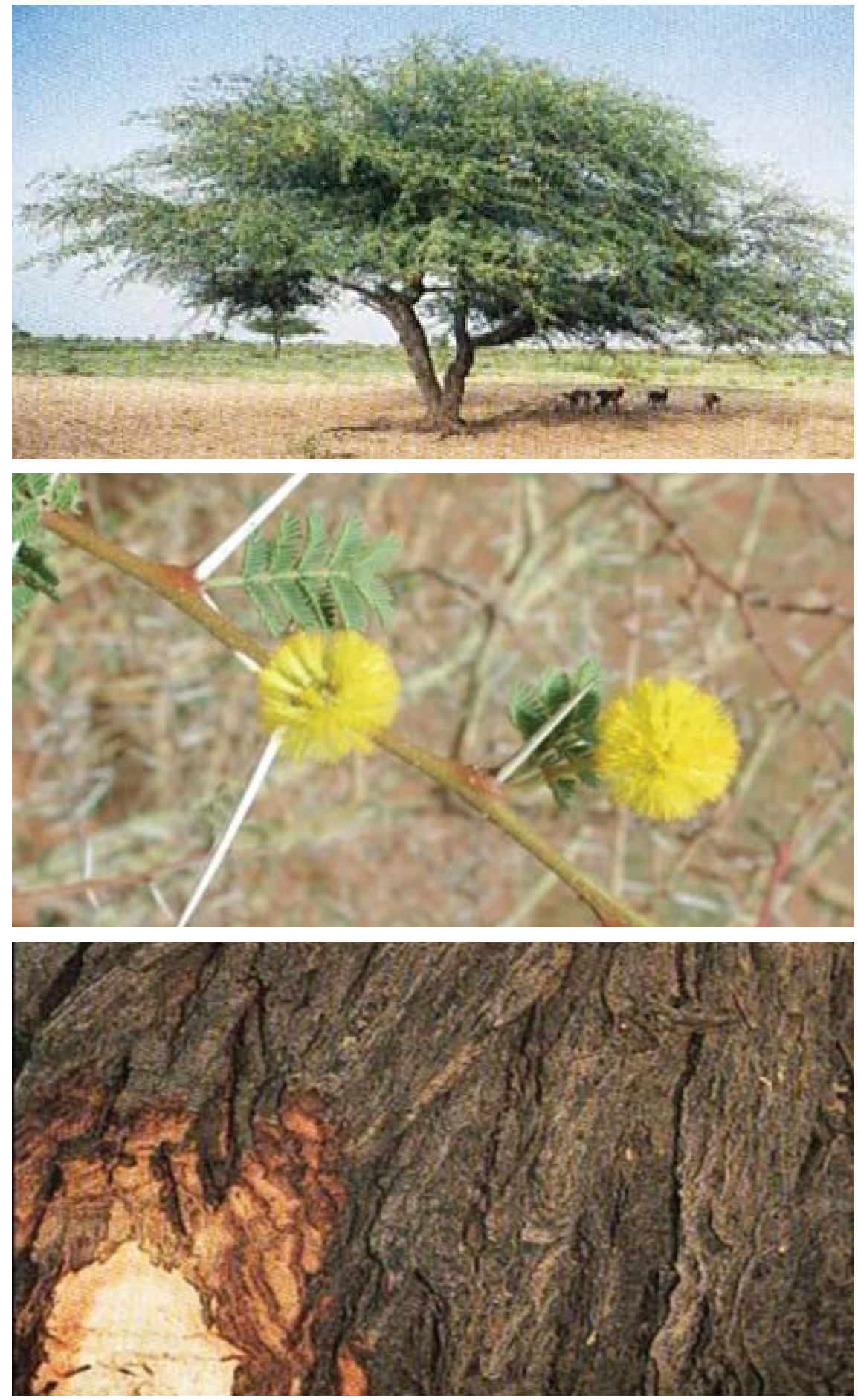

Photo 4. Acacia seyal (@ DTA et CIFOR). 

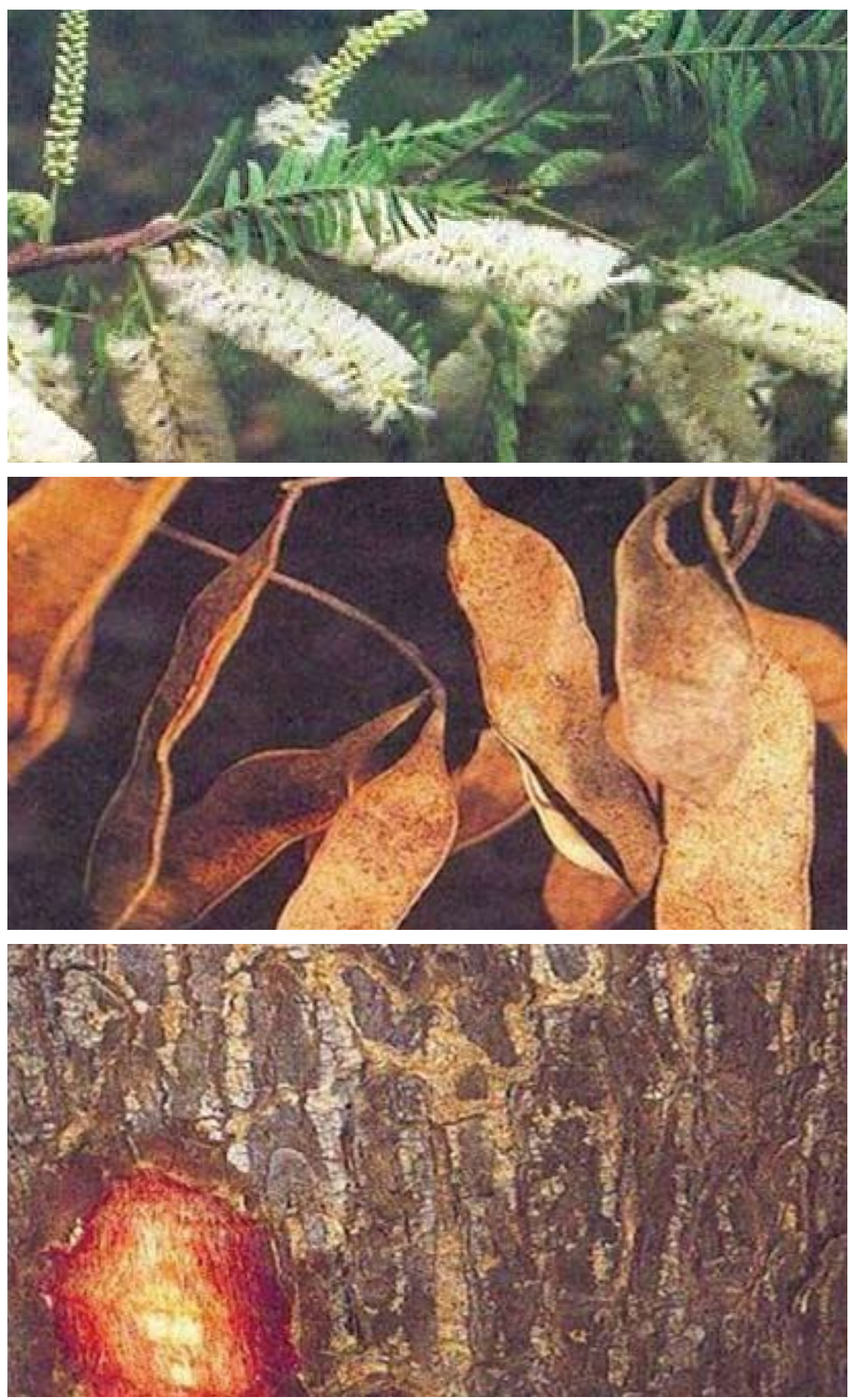

Photo 5. Acacia dudgeoni (@ DTA). 


\subsubsection{La gommeraie}

Une gommeraie est une plantation ou un boisement d'arbres producteurs de gomme. Ce boisement peut être naturel ou artificiel. Il existe deux méthodes de mise en place d'une gommeraie : la méthode naturelle et la méthode artificielle. Le peuplement ou boisement sera dit naturel s'il provient de la méthode naturelle, ou artificiel s'il a été mis en place par voie artificielle.

\subsubsection{Classification des gommes}

On retrouve généralement sur le marché au Burkina, les gommes issues essentiellement de quatre espèces à savoir Acacia senegal, Acacia laeta, Acacia seyal, Acacia dudgeoni.

Suivant leur aspect au toucher, on distingue deux types de gomme :

- les gommes dures, qui ne cèdent pas sous la pression des doigts;

- les gommes friables, qui seffritent et tombent en lambeaux sous la pression des doigts.

Il existe d'autres classifications sur le plan commercial ou en fonction des propriétés physico-chimiques des gommes.

Certains appellent gomme arabique ou gomme dure, la gomme produite par Acacia senegal et les espèces qui lui sont voisines à savoir Acacia laeta et Acacia dudgeoni. Plusieurs auteurs (Djiré et al., 2000 ; Fagg et Allison, 2004) confirment en effet que les propriétés physico-chimiques des gommes de ces deux espèces sont similaires à celles de la gomme produite par Acacia senegal.

Différenciation suivant les usages : Les différents usages des gommes dépendent en réalité de leurs propriétés physico-chimiques mais selon le classement de la JECFA (1999), est appelée gomme arabique la gomme dure produite par Acacia senegal et la gomme friable issue de Acacia seyal.

Les autres espèces gommifères produiraient des gommes représentant un intérêt économique moins important que la gomme arabique quoique Bognounou (2000) les considère comme « les ressources du futur». 


\subsubsection{Les résines}

La résine est l'exsudat de certaines espèces telles que Comiphora africana, Daniella oliveri, Boswellia dalzielli, utilisé pour la production de la myrrhe, de l'encens, du parfum.

Les gommes diffèrent des résines par leur comportement face à l'eau et aux alcools. La gomme se dissout dans l'eau tandis que les résines sont très sensibles aux solutions d'alcools.

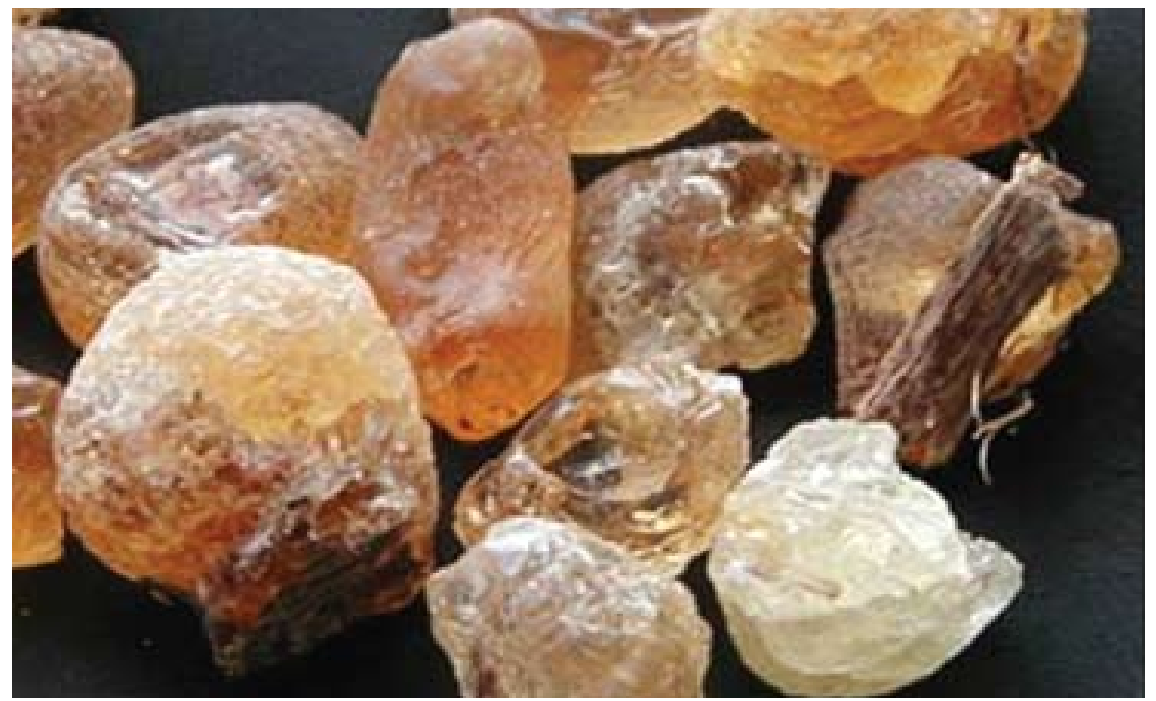

Photo 6. Gomme d'Acacia senegal (@ DiFor).

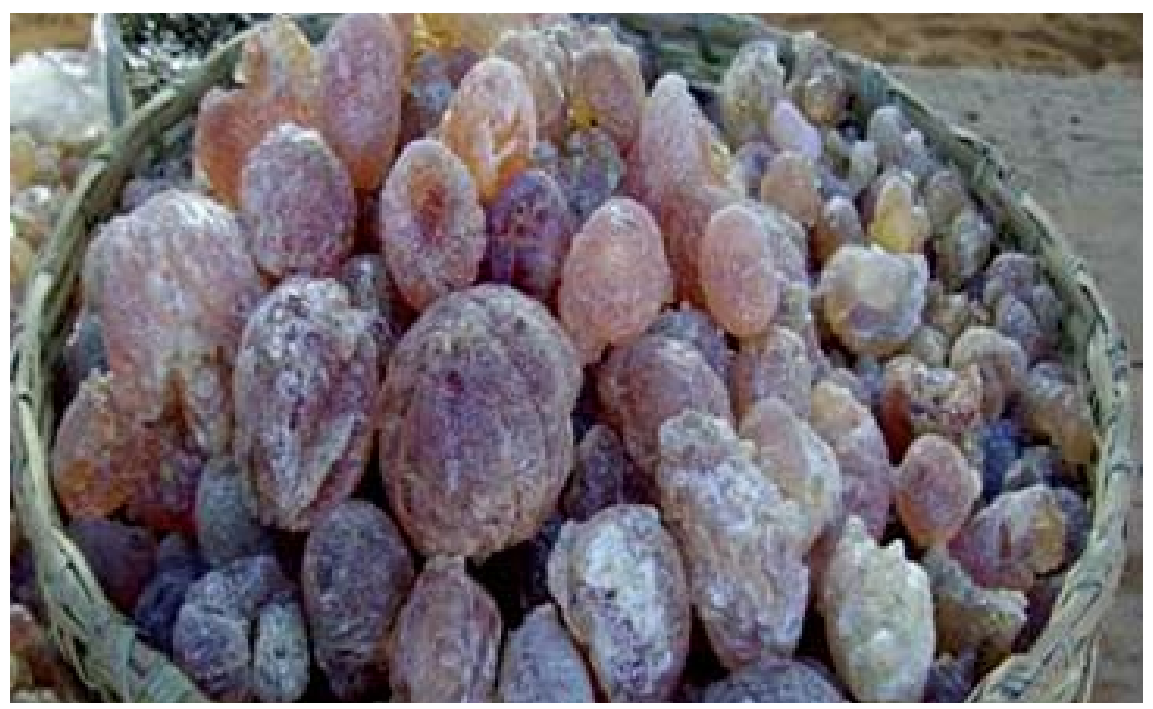

Photo 7. Nodules de gomme d'Acacia prêtes pour la vente (@ DiFor). 


\subsection{Utilité des espèces gommifères et de la gomme}

\subsubsection{Utilité des acacias gommiers}

Lacacia gommier est par excellence une espèce à usages multiples.

\section{Usages au plan agricole}

Les acacias gommiers sont des Mimosaceae et ont de ce fait la propriété de fixer l'azote atmosphérique. Ils ont ainsi la capacité de reconstituer les sols à la fois grâce à l'abondance de leur biomasse végétale et à leur aptitude à mobiliser l'azote atmosphérique. Il se forme donc un processus cyclique de production, d'absorption et de décomposition déléments minéraux qui enrichissent le sol dans l'environnement immédiat de l'arbre, favorisant ainsi le bon développement des cultures.

Les acacias gommiers, en particulier Acacia senegal et Acacia laeta, sont utilisés au Burkina Faso dans les travaux de DRS/CES (défense et restauration des sols/conservation des eaux et des sols).

Les plants d'Acacia laeta et d'Acacia senegal ont effectivement été utilisés pour la revégétalisation des terres dénudées (appelées «zipélé » en langue locale mooré) après sous-solage aux rippers de bull ou à la charrue Delfino dans le cadre de plusieurs projets dont le projet régional «Forêts et sécurité alimentaire en Afrique Sahélienne »(1992-2000), le projet " front de terre "(1998-2004), le projet "FAO - Opération acacia » (2004-2006) et actuellement dans le cadre du projet " programme de lutte contre l'ensablement dans le bassin du fleuve Niger (PLCE/ABN) » pour la récupération des terres dégradées.

En outre la mise en défens, renforcée par le système de demi-lune, est un procédé connu des populations de la région de Ouahigouya pour la reconstitution du couvert végétal à partir d'Acacia senegal ou d'Acacia laeta en vue de la valorisation agricole des terres dénudées. 


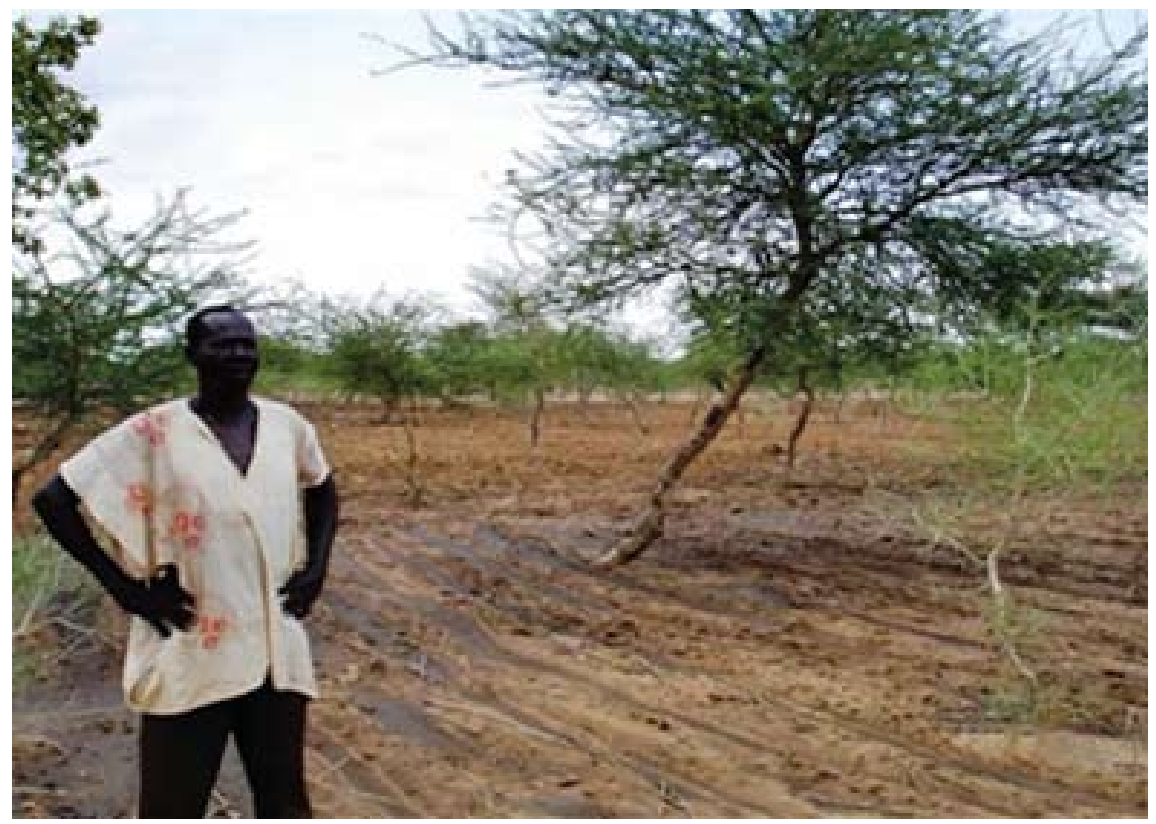

Photo 8. Champ de culture sous peuplement d'Acacia (@ DiFor).

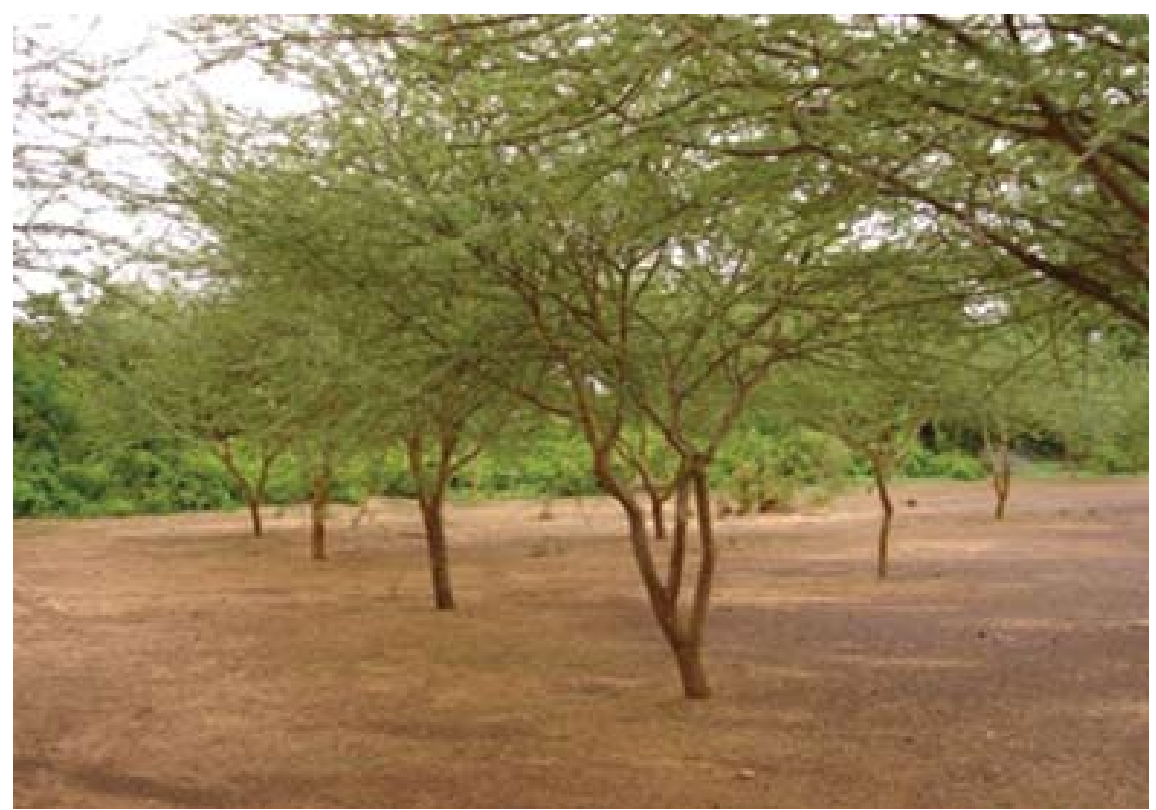

Photo 9. Reconstitution du couvert végétal à partir de plantations d'Acacia senegal (@ DiFor). 
- Alimentation des animaux : Dans les régions du Sahel et du Nord du Burkina, les Acacias constituent 95\% du pâturage aérien. Les feuilles et les gousses des principaux gommiers sont particulièrement appréciées par le bétail.

- Production du miel : Les acacias sont des espèces mellifères et par conséquent, fournissent aux abeilles la matière première pour la production de miel.

- Bois de service et d'énergie : Le bois des acacias est beaucoup utilisé dans les régions du Sahel et du Nord du Burkina Faso comme bois de service (enclos) et comme bois dénergie (bois de chauffe, charbon de bois).

- Confection de haies vives : Les branches épineuses des acacias sont utilisées pour confectionner les haies mortes en vue de protéger les parcelles de cultures ou pour délimiter les enclos servant d'abris aux animaux domestiques.

- Confection de haies vives : Les plantes d'acacia peuvent servir de haies vives pour la protection des plantations, des champs, des pépinières et autres formes de cultures à cause de leurs épines qui empêchent le passage des animaux.

- La pharmacopée traditionnelle : Les racines, les feuilles, l'écorce d'Acacia senegal et d'A. laeta sont utilisées dans la médecine traditionnelle pour soigner différents maux.

- Artisanat : Le bois des gommiers est utilisé pour la fabrication d'objets d'art.

- La production de gomme : C'est la fonction la plus importante de l'acacia gommier.

En résumé, l'Acacia gommier est une espèce offrant de nombreuses fonctions utiles au producteur pour l'amélioration de son activité principale qui est la culture des céréales et pour la génération de revenus. Il contribue également à la lutte contre la désertification.

\subsubsection{Utilité de la gomme}

\section{Utilisations traditionnelles de la gomme}

$\mathrm{Au}$ Burkina Faso, les gommes sont connues et exploitées pour de multiples usages, aussi bien domestiques que dans de petites activités de production. Elles interviennent dans la fabrication dencre, de peinture, dans la réalisation de crépi, dans l'empesage des tissus, etc. Dans la pharmacopée 
traditionnelle, elles sont utilisées pour les soins de différents maux tels que la dysenterie, les ballonnements de ventre, les rhumes, les toux, les maux de dents. Elles interviennent également dans la préparation de certaines infusions pour les soins vétérinaires.

\section{Utilisations industrielles de la gomme}

La gomme est un produit insipide, inodore et non toxique. Elle constitue une matière première qui rentre dans la fabrication ou la préparation de plusieurs produits alimentaires et industriels.

Tableau 1 : Différents usages des gommes.

\begin{tabular}{|c|c|}
\hline $\begin{array}{l}\text { Secteurs } \\
\text { d'utilisation } \\
\text { des gommes }\end{array}$ & Principales applications \\
\hline \multicolumn{2}{|l|}{ Secteur alimentaire } \\
\hline Confiserie & $\begin{array}{l}\text { Pastillage, dragéification, pâte à mâcher, } \\
\text { chewing-gum, etc. }\end{array}$ \\
\hline \multirow{2}{*}{ Boisson aromatique } & Soda, sirop, boissons \\
\hline & Stabilisation, mousse de bière, etc. \\
\hline Produits aromatiques & $\begin{array}{l}\text { Arômes en poudre, troublants en poudre, } \\
\text { boissons instantanées }\end{array}$ \\
\hline OEnologie & Suspension de tanin, stabilisation des vins \\
\hline Produits alimentaires & $\begin{array}{l}\text { Enrobage de fruits secs, sauces, } \\
\text { condiments, glaçage biscuits, gâteaux, } \\
\text { desserts, etc. }\end{array}$ \\
\hline \multicolumn{2}{|c|}{ Secteur pharmaceutique } \\
\hline Pharmacie & $\begin{array}{l}\text { Pâtes pectorales, vitamines en poudre, } \\
\text { dragées, pilules, sirops, etc. }\end{array}$ \\
\hline \multicolumn{2}{|l|}{ Secteur divers } \\
\hline $\begin{array}{l}\text { Lithographie, produits } \\
\text { offset }\end{array}$ & $\begin{array}{l}\text { Protection des plaques, offset, anti- } \\
\text { maculateur, mouillage de rouleaux }\end{array}$ \\
\hline Colles & $\begin{array}{l}\text { Colle de bureau, timbres, enveloppe, } \\
\text { papier gommé }\end{array}$ \\
\hline Cosmétique & Savon liquide et lotions \\
\hline Divers & $\begin{array}{l}\text { Fonderie, céramique, pyrotechnie, } \\
\text { explosifs, insecticides, pesticides, } \\
\text { industrie textile, etc. }\end{array}$ \\
\hline
\end{tabular}




\section{Module II \\ Aménagement et gestion d'une gommeraie}

T orsque le peuplement de gommeraie est mis en place par la main de l'homme, il est dit artificiel. Dans le cas contraire, on parle d'un peuplement naturel. Ces deux méthodes de mise en place de la gommeraie ont des exigences qui peuvent garantir la qualité de la production de la gomme.

\subsection{La méthode naturelle}

Le boisement ou peuplement naturel est issu de la régénération naturelle. Cette régénération naturelle peut être assistée on parle de RNA. Dans ce cas, la régénération a été d'une manière ou d'une autre facilitée par l'homme. Il existe plusieurs cas de figure :

\subsubsection{Jeunes plants de gommiers épargnés volontairement dans le champ lors des labours et entretenus}

Il s'agit ici d'intégrer le gommier dans les champs de culture pour constituer un système agroforestier. Les étapes à suivre sont les suivantes :

- Déterminer la parcelle dans laquelle on veut appliquer la RNA ;

- Compter le nombre de pieds adultes dans la parcelle ;

- Identifier, repérer, compter les jeunes pousses de gommiers dans la parcelle. Les jeunes pieds sont repérés par peinture ou à l'aide d'un piquet implanté au pied du plant ;

- Protéger les jeunes plants ainsi repérés. 
Le nombre optimum de pieds de gommiers à maintenir dans un champ d'un hectare en système agroforestier est de 25 pieds adultes et 60 à 80 pieds jeunes.

La régénération assistée a l'avantage dêttre à la portée du paysan pour installer sa gommeraie. Elle nest pas onéreuse car on na pas recours aux plants produits en pépinière qui coûtent chers. La technique est très simple et facile à vulgariser.

\subsubsection{Mise en défens}

La mise en défens est l'ensemble des techniques et des mesures de protection mises en ouvre dans une parcelle pour permettre la réinstallation de la couverture végétale et la reconstitution du sol. Les techniques couramment associées surtout au nord et dans la région sahélienne sont le paillage, le sous-solage avec la charrue Delfino, le zaï forestier ${ }^{4}$, la protection intégrale de la parcelle.

La mise en défens permet la réinstallation de la gommeraie, la récupération des terres dégradées et leur remise en culture.

\subsubsection{Pacage et mise en stabulation des animaux dans une aire définie}

On fait brouter les gousses du gommier par les animaux. Les graines consommées subissent un prétraitement dans l'appareil digestif de l'animal. Les graines sont ensemencées à travers les bouses déposées directement sur le terrain par les animaux ou à l'issue de l'épandage du fumier dans le champ.

D’une manière générale, le vent et les animaux constituent les principaux agents de dissémination des graines des gommiers.

\footnotetext{
${ }^{4}$ Le zaï forestier est un poquet de 30 à $40 \mathrm{~cm}$ de diamètre et 15 à $20 \mathrm{~cm}$ de profondeur avec la terre excavée vers l'aval du poquet en forme de croissant, partiellement rempli de fumier, au centre duquel on sème dès les premières pluies une graine d'espèces forestières (Acacia senegal en l'occurrence) et à la périphérie des graines de céréales. A la récolte des céréales, les tiges sont coupées à une hauteur d'environ $1 \mathrm{~m}$ au dessus du sol pour protéger larbre du vent et du bétail.
} 


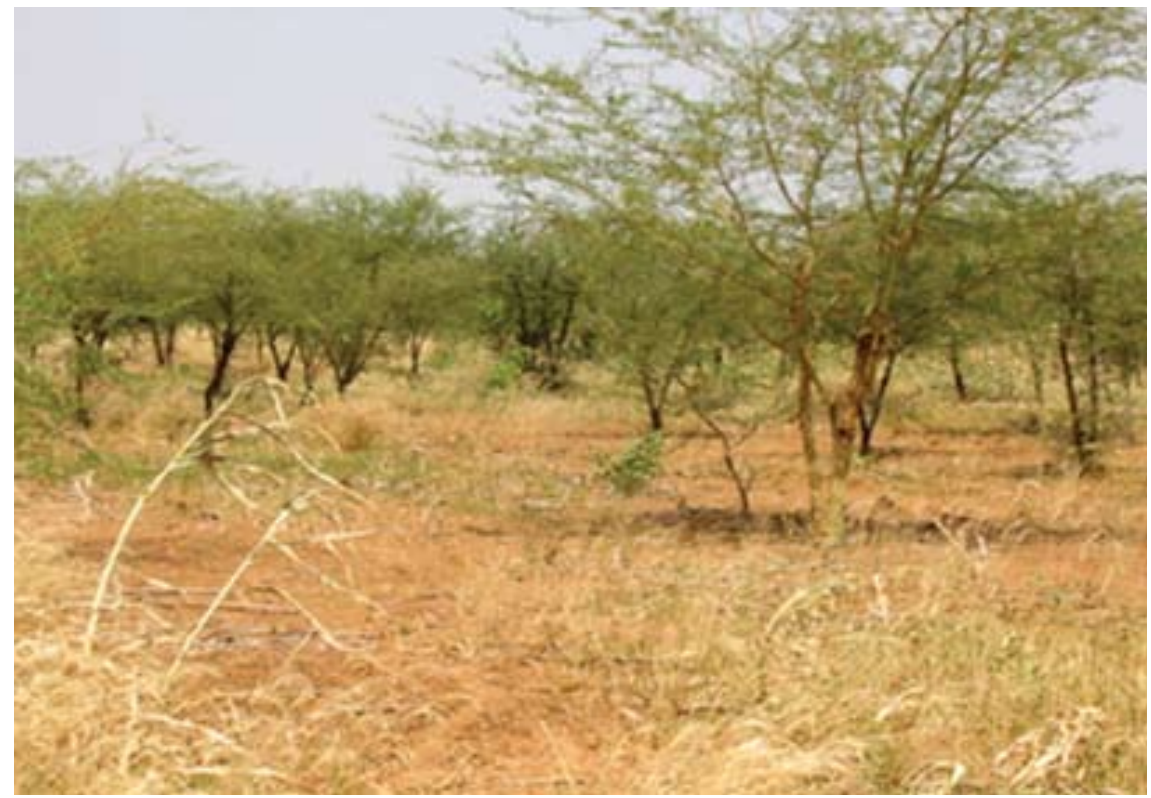

Photo 10. Peuplement naturel d'Acacia à Bogandé (@ DiFor).

\subsection{Méthode artificielle de réalisation de gommeraie}

La gommeraie est dite artificielle lorsque le peuplement a été mis en place soit par plantation à partir de plants élevés en pépinière, soit par semis direct.

\subsubsection{Plantation de gommeraie}

Si la première vocation de la gommeraie est économique à travers la production de la gomme destinée notamment au marché international, le choix de l'espèce est alors capital. L'espèce à privilégier dans ce cas est Acacia senegal parce que produisant la gomme la plus recherchée et la plus cotée.

\section{Les semences}

Lapprovisionnement en semences de bonne qualité est primordial dans la chaîne de production des plants pour la réalisation artificielle d'une gommeraie. Lacquisition des semences peut se faire de deux manières :

- auprès du Centre National de Semences Forestières (CNSF) ou des services forestiers déconcentrés ;

- en produisant soi-même les graines. 


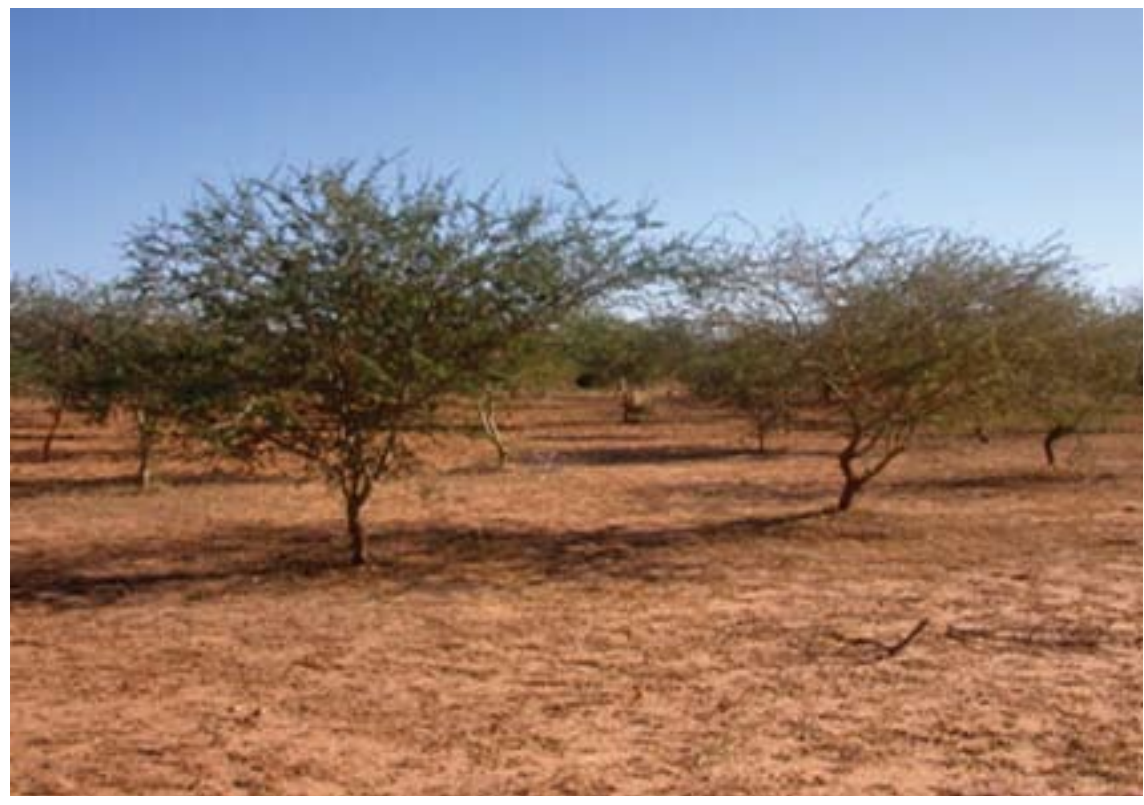

Photo 11. Une gommeraie artificielle d'Acacia senegal à Mani (๑ DiFor).

Les principales opérations pour produire des semences de bonne qualité sont les suivantes :

- identification des gommiers bien portants et producteurs de gomme de bonne qualité comme porte graines ;

- récolte des fruits bien mûrs sur des pieds sains, distants d'au moins $100 \mathrm{~m}$ les uns des autres. Si le peuplement a été mis en place avec des semences certifiées, la récolte se fera sur chaque pied;

- séchage ;

- extraction des graines à la main ou par pilage ;

- vannage et tri ;

- conservation des semences jusquà leur utilisation. Les graines sont très sensibles aux bruches et autres prédateurs. Elles peuvent être conservées en utilisant les insecticides chimiques vendus sur le marché ou des produits naturels extraits de plantes.

L'insecticide naturel le plus connu est l'huile de graines de neem (Azadirachta indica). Le dosage recommandé pour son utilisation est de trois (3) $\mathrm{ml}$ par kilogramme de graines à conserver, soit léquivalent d'une capsule de bouteille de bière. 
Il existe d'autres méthodes traditionnelles de conservation des semences notamment avec la cendre.

La période de récolte des semences d'Acacia senegal se situe entre le mois de novembre et le mois de décembre. Pour éviter que les graines ne soient attaquées par les insectes sur l'arbre, il faut les récolter dès qu'elles commencent à changer de couleur (du vert au jaune brun).

\section{La production des plants}

\section{- Le site de production}

o Les plants sont élevés en pépinière implantée et aménagée conformément aux règles techniques connues;

o Site implanté sur un bon sol, bien protégé, à proximité des habitations pour faciliter la surveillance ;

o Disponibilité d'un point d'eau et de matériel de pépinière.

\section{- Le conteneur}

Les sachets plastiques sont les mieux indiqués. Mais en raison de leur coût élevé, certains producteurs ont recours à des matériaux de récupération tels que les sachets d'eau. Une autre technique consiste à utiliser des mottes de terre confectionnées à l'aide d'argile mélangée à la paille.

\section{- Le prétraitement des semences}

Le prétraitement est une opération qui permet la levée de l'inhibition tégumentaire ou dormance. Il permet d'améliorer la germination des graines. Le meilleur traitement des graines d'Acacia senegal est l'ébouillantage suivi du trempage dans l'eau pendant $24 \mathrm{~h}$.

\section{- Les semis et les soins à donner aux plantules}

Les semis dans les pots commencent en mars. Limportant est de programmer la production de sorte que la plantule ait séjourné au moins trois mois dans la pépinière avant dêtre plantée. Les soins à apporter après la germination sont les suivants :

o le démariage, le désherbage dans le pot, le binage ;

- le déplacement des pots tous les 15 à 20 jours afin que les racines ne pénètrent pas dans le sol. Lors du déplacement des pots, tailler les racines qui ont pu traverser le pot ;

- la surveillance sanitaire des plantules et les traitements phytosanitaires chaque fois que de besoin ; 
o l'arrosage matin et soir;

o l'installation d'une ombrière pour protéger les plantules pendant 2 semaines après la germination.

\section{Plantation et entretien des plants}

\section{- La préparation et le transport des plants pour la plantation}

Le transport des plants est une opération délicate. Les précautions suivantes sont à prendre avant la sortie des plants de la pépinière :

o bien arroser les plants la veille de la sortie ;

o manipuler les pots avec soin lors du chargement et du déchargement ;

o déposer les plants à l’ombre si la plantation doit intervenir plus tard et au besoin les arroser.

\section{- Le choix du site de plantation}

Les gommiers seront plantés dans les zones correspondant à leur aire naturelle de répartition en tenant compte de leur préférence quant aux types de sols et à la topographie. Laire naturelle ou potentielle d'Acacia senegal et d'Acacia laeta est définie sur la carte ci-dessous.

\section{- La préparation du terrain}

o Le défrichement du terrain

Il se fait manuellement avec les outils aratoires du terroir ou mécaniquement. Au Burkina Faso, le sous-solage aux rippers de bull, à la charrue Delfino ou à la charrue Treno donne de très bons résultats de plantation.

o Le sous-solage à la charrue Delfino

La charrue Delfino est la plus utilisée actuellement dans les projets de développement au Burkina. Elle présente des limites et des exigences dans son utilisation. Les conditions favorables sont :

- sols lourds, argileux à sablo-argileux et argilo-gravillonnaire, compacts à très compacts et /ou pierreux;

- pente de terrain comprise de 0 à $10 \%$;

- profondeur du sol supérieure à $80 \mathrm{~cm}$;

- texture du sol pas trop sableuse;

- absence de cuirasse ou de roche sub-affleurante ;

- absence de souche. 


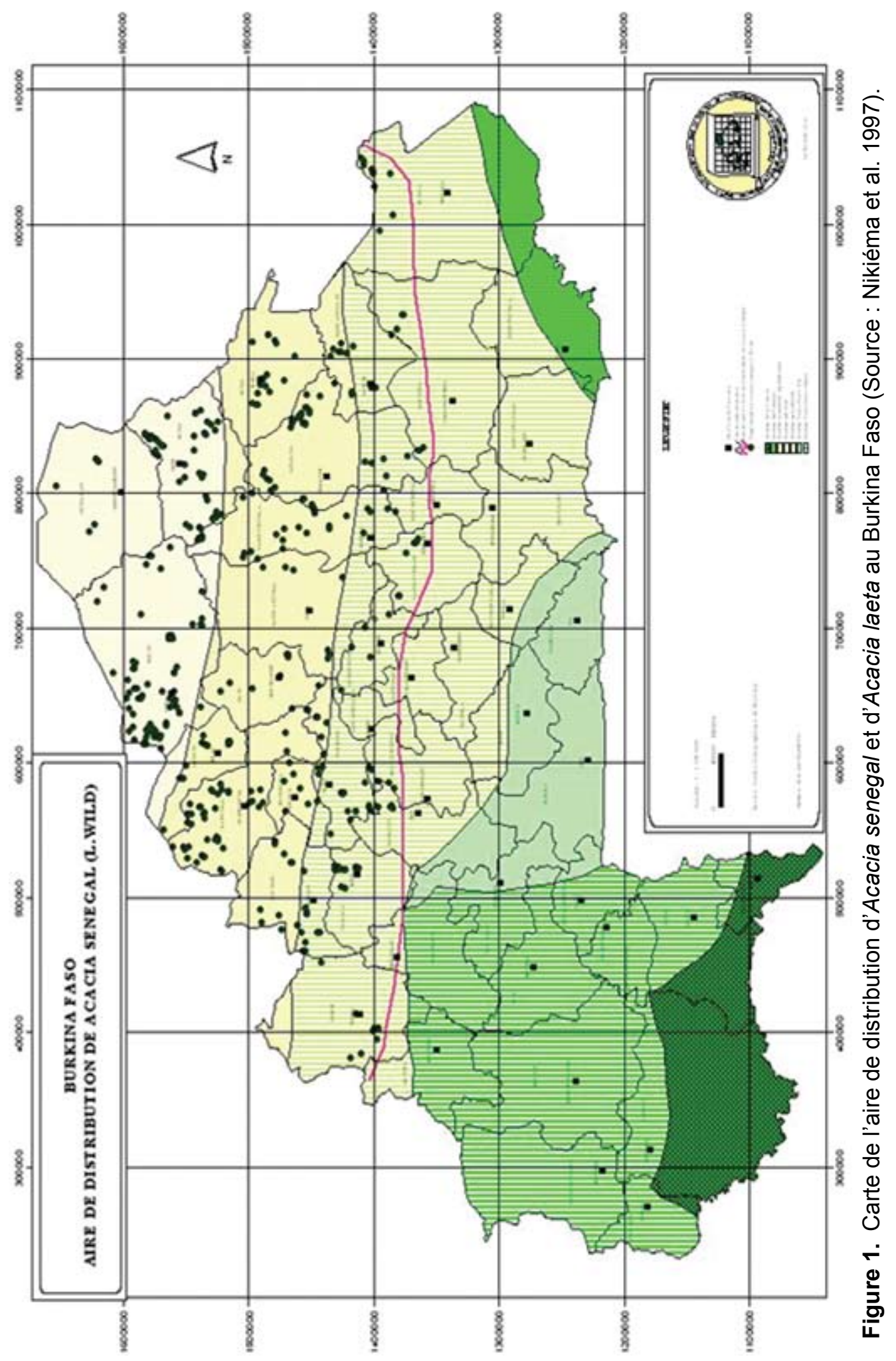


Le sous-solage est réalisé perpendiculairement à la pente du terrain. Les dimensions de la demi-lune confectionnée sont les suivantes :

- longueur de 4, 5 - $5 \mathrm{~m}$;

- une largeur de $0,8-1 \mathrm{~m}$;

- une profondeur de $0,4-0,6 \mathrm{~m}$.

Le nombre de demi-lunes par hectare varie en fonction de l'écartement entre les lignes lui-même lié à la pluviométrie. Les prescriptions du concepteur sont les suivantes:

- écartement de 3 à $4 \mathrm{~m}$ pour une pluviométrie comprise entre 300 et $350 \mathrm{~mm} / \mathrm{an}$, soit 470 à 350 demi-lunes/ha ;

- écartement de 5 à $7 \mathrm{~m}$ pour une pluviométrie de $200 \mathrm{~mm} / \mathrm{an}$, soit 280 à 142 demi-lunes/ha.

Lécartement de $5 \mathrm{~m}$ entre les lignes est le plus couramment adopté au Burkina Faso.

○ Le sous-solage aux rippers de bull

Le sous-solage croisé est une technique de préparation mécanique du sol destinée à la plantation des arbres. Elle a été utilisée pendant un certain temps avec succès dans de nombreux chantiers de

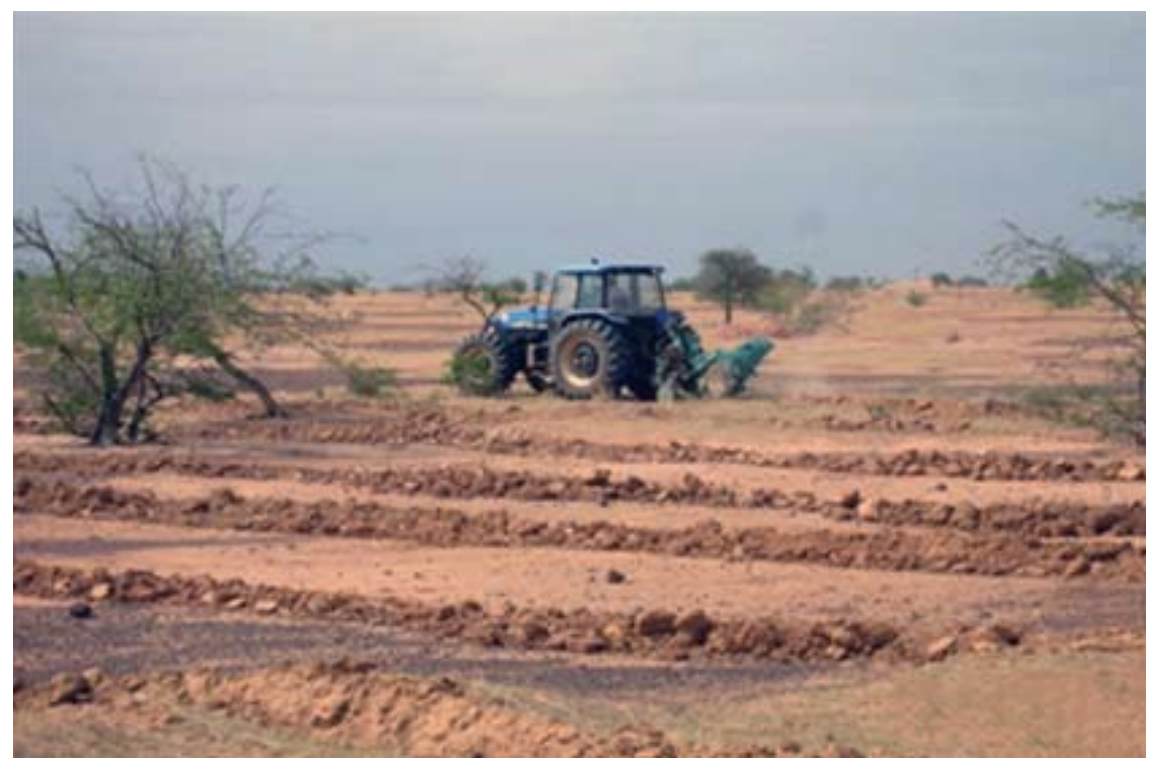

Photo 12. Sous-solage à la charrue Delfino d'une parcelle de plantation à Tougouri (@ DiFor). 
reboisement telles la plantation industrielle de Wayen et les plantations du projet « Front de terre » à Louda, Bangaye, Boulsa. Le sous soulage croisé est réalisé avec un Bulldozer D6 ou D7 avec pour conséquence un coût élevé des travaux, ce qui constitue un handicap pour les producteurs.

La technique consiste à réaliser un labour croisé à l'aide du ripper du Bull de trois dents d'une profondeur de 60 à $70 \mathrm{~cm}$ à un écartement de $5 \times 5$ mètres ou $6 \times 6$ mètres. Les intersections des sillons perpendiculaires constituent les emplacements des plants. Cette technique remplace la trouaison pour les vastes superficies à reboiser. Elle peut se réaliser après le piquetage ou par simple jalonnement.

\section{- La plantation}

La plantation comporte les opérations suivantes :

o Le piquetage

Le piquetage consiste à matérialiser les trous des plants en implantant des piquets. Le piquetage définit la densité de la plantation. Le piquetage permet de planter en ligne équidistante. Lécartement entre deux emplacements de piquet est défini à l'aide soit d'une ficelle ou d'un bâton pré dimensionné, d'un instrument de mesure ou par les pas. En fonction des spéculations assignées à la parcelle, (exploitation exclusive de gomme ou en association avec des cultures), on adoptera une plantation dense ou peu dense.

Les écartements appliqués sont variables :

- lécartement de $4 \mathrm{~m} \mathrm{X} 4 \mathrm{~m}$ soit une densité de 625 plants par hectare est le plus courant au Burkina Faso dans les campagnes de reboisement ;

- lécartement de $5 \mathrm{~m} \mathrm{X} 5 \mathrm{~m}$ pour une densité de 400 arbres par hectare permet de pratiquer dans les parcelles les cultures basses (arachide, niébé) ;

- lécartement de $8 \mathrm{~m} \mathrm{X} 8 \mathrm{~m}$ pour une densité de 156 arbres par hectares est adopté quand la parcelle doit porter également des cultures de hautes tiges (mil, sorgho, maïs). 
- La trouaison

Les trous sont creusés à l'emplacement des piquets. Lors du creusage, la terre de la couche supérieure, généralement plus riche en matière nutritive et appelée terre noire est séparée de la terre issue des couches en profondeur, d'aspect rouge, plus pauvre en matières organiques. Les trous sont creusés aux dimensions de $60 \mathrm{~cm}$ de profondeur $\mathrm{x} 60$ $\mathrm{cm}$ de diamètre.

o Rebouchage des trous

Deux cas de figure sont possibles :

$\mathbf{1}^{\text {er }}$ cas : Les trous sont rebouchés sur le champ, après la trouaison. On met au fond du trou la terre dite noire enlevée en premier lieu lors de la trouaison et ensuite la terre rouge. Les trous ne doivent pas être remplis complètement; on laisse au moins $5 \mathrm{~cm}$ pour constituer une petite cuvette.

$2^{\text {ème }}$ cas : Les trous sont rebouchés au moment de la plantation.

- Mise en terre des plants

La plantation se déroule quand la saison hivernale s'est bien installée soit de juillet à mi-août. La plantation est réalisée en période de basse température, soit le matin, soit le soir ou alors par temps couvert. La mise en terre des plants suit les étapes suivantes :

- réaliser au centre du trou préalablement rebouché, une ouverture aux dimensions du sachet plastique; reboucher partiellement le trou de sorte que la partie non rebouchée corresponde aux dimensions du sachet plastique ;

- découper le fond du pot avec un objet tranchant en maintenant le plant couché ; cette opération est nécessaire car elle permet de supprimer les racines qui se sont enroulées dans le fond du pot et facilite ainsi la croissance racinaire ;

- inciser le pot sur toute sa hauteur ;

- déposer délicatement le plant toujours entouré du sachet plastique dans le trou matérialisé au préalable ;

- une fois le plant dans le trou, déposer un peu de terre autour du pot pour le maintenir droit et enlever l'enveloppe plastique en la tirant vers le haut ;

- reboucher complètement le trou en prenant soin de bien tasser la terre autour du plant tout en créant une légère dépression pour retenir l'eau. 
Il faut cependant préconiser une séance de ramassage des sachets après la mise en terre des plants afin déviter la pollution de lenvironnement ou leur consommation par les animaux.

o Le regarnissage

Le regarnissage est une opération de plantation qui seffectue une ou deux années après la plantation proprement dite. Il a pour but de remplacer les plants morts ou mal portants. Le regarnissage permet de suivre et dévaluer en même temps le comportement des plants afin de prendre les mesures correctives.

\subsubsection{Le semi direct}

Linstallation de la gommeraie peut se faire aussi par semis direct des graines. Les graines sont ensemencées en début de saison hivernale soit à la volée, soit dans des poquets préalablement préparés, disposés en lignes, à écartement retenu par le producteur. Chaque poquet recevra 2 à 3 graines. On procédera ultérieurement à un démariage après la germination.

\subsection{L'entretien de la gommeraie}

L'entretien de la gommeraie, qu'elle soit naturelle ou artificielle, comporte les travaux suivants :

- La protection contre les animaux. Il faut soustraire les plantules des dents du bétail pendant au moins trois ans après leur mise en terre ou leur germination. La région sahélienne étant une zone délevage par excellence, la protection des plants contre les animaux constitue un défi. Pour cela, il est conseillé de privilégier les petites surfaces qui sont plus faciles à surveiller. Les grandes superficies de plantation devraient faire l'objet d'une attention particulière par des actions de gardiennage qui seront rendues possibles par une organisation communautaire fondée sur la volonté des membres.

- La lutte contre les termites. Les termites constituent une des principales causes de mortalité des plants nouvellement mis en terre. On peut recourir, lorsque la situation l'exige et que les moyens le permettent, aux traitements insecticides. Mais les mesures préventives sont toujours préférables, comme l'utilisation de plants vigoureux, et si nécessaire, le nettoyage des alentours du trou et du site. 

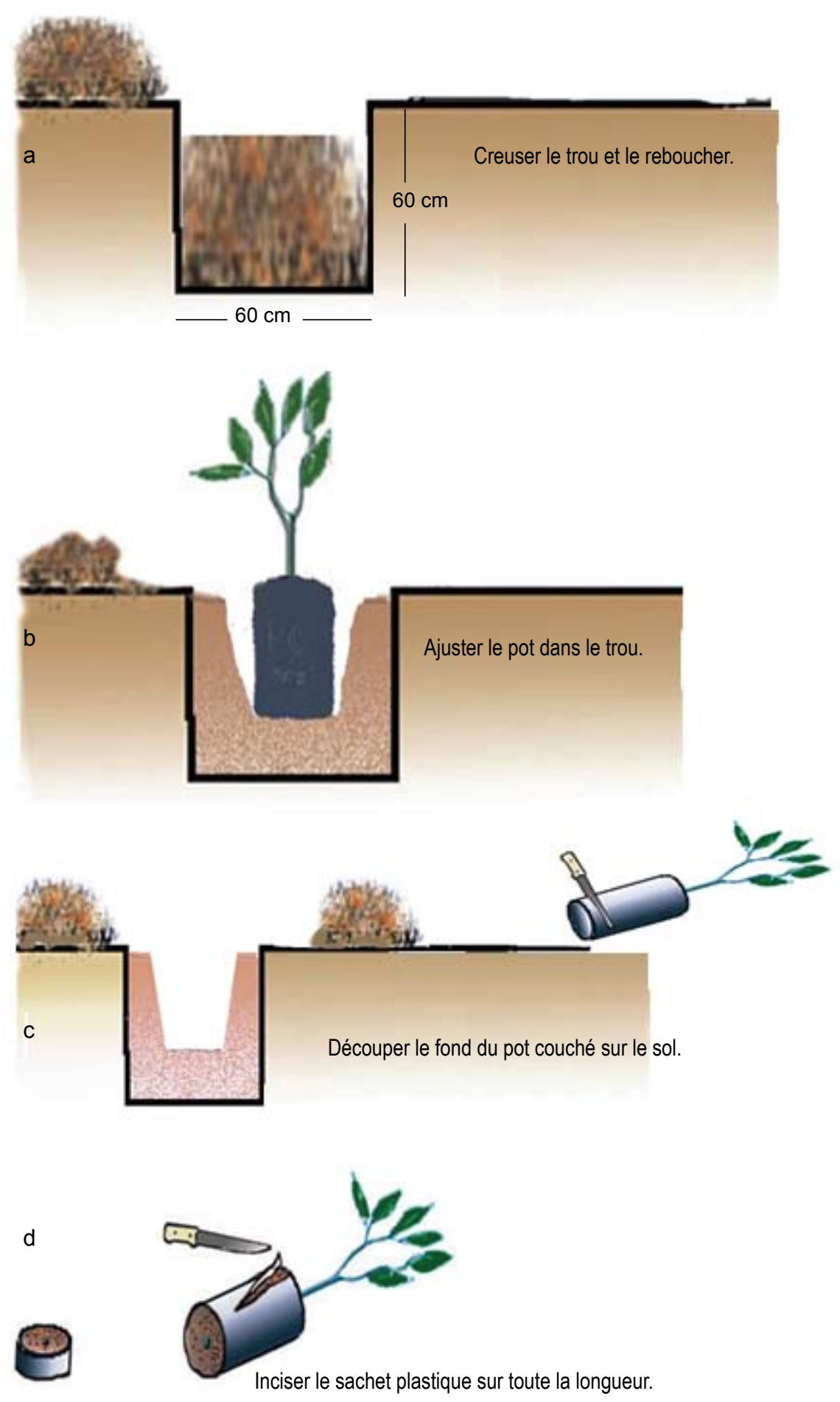

partie découpée. 

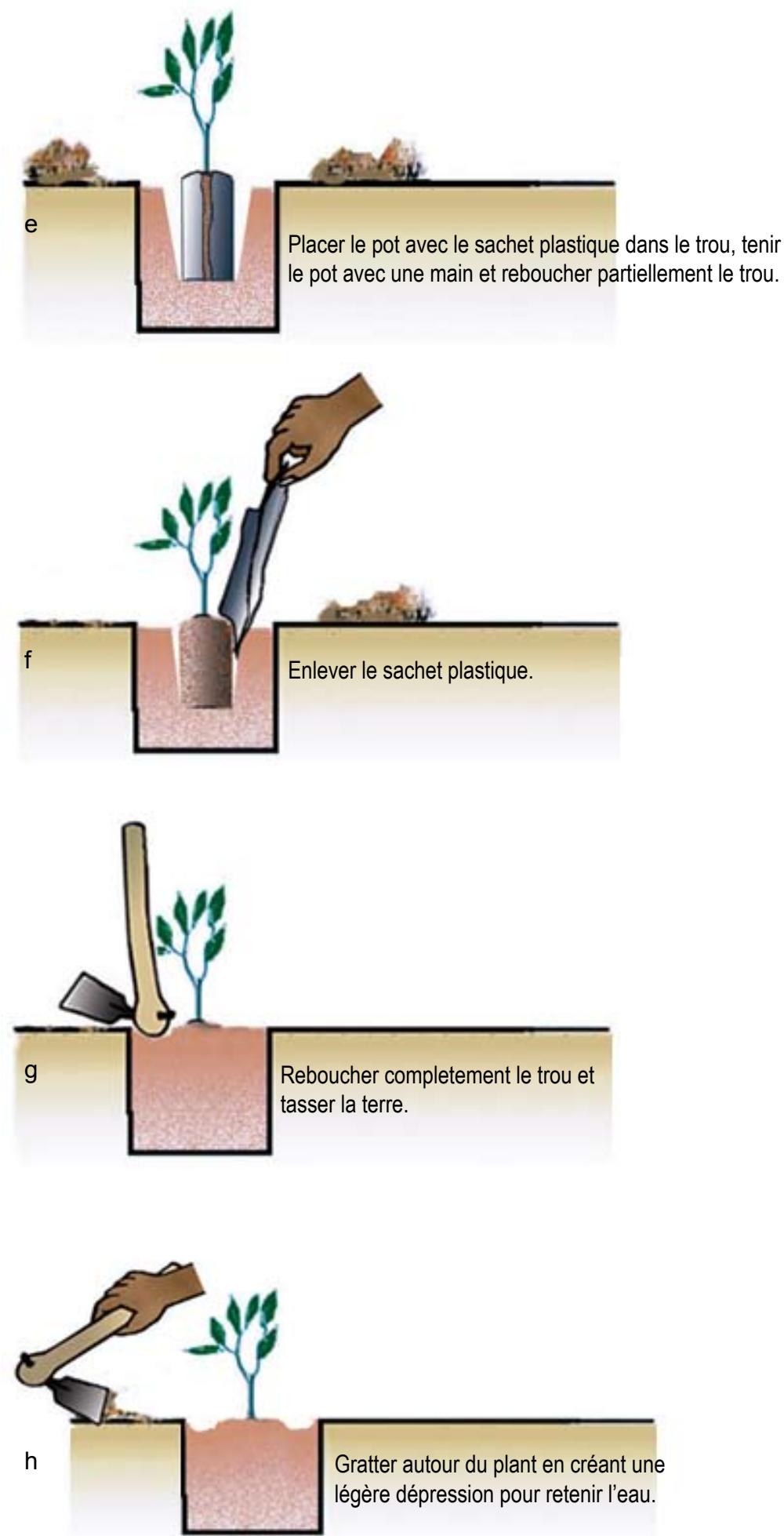

Figure 2. Opérations de mise en terre d'un plant en pot (Source : Manuel de foresterie villageoise, DiFor). 
- Le désherbage. Le désherbage du site n’est pas toujours nécessaire, surtout dans la région sahélienne où l'herbe est recherchée pour le fourrage. Le tapis herbacé constitue également une couverture végétale qui contribue à freiner lérosion et est le premier indicateur de récupération des sols dénudés.

- La coupe sanitaire. Couper les branches mortes ou attaquées. Supprimer les branches parasitées par le Thapinantus.

- Lélagage. Supprimer les branches basses qui pourraient gêner les opérations de saignée.

- L’application des techniques de DRS/CES. Ce sont tous les procédés qui visent à récupérer l'eau pour la mettre à la disposition de la plante ou à ameublir le sol pour faciliter la croissance racinaire de la plantule : le système de demi-lune, les dispositifs de cuvette, la méthode Zaï, la mise en défens, le sous-solage à la charrue Delfino, etc.

- Les cultures intercalaires. Elles sont pratiquées dans les parcelles où les peuplements sont âgés de 0 à 5 ans. Le système agroforestier sous les gommiers améliore le rendement des cultures et favorise la croissance des plants de gommier. L'utilisation des cultures sera préalablement établie par lécartement préconisé au moment de la trouaison. Les cultures de haute taille (mil, sorgho, maïs, etc.) exigent un écartement plus grand que celles qui sont plus basses (Arachide, niébé, poids de terre).

\subsection{Gestion de la gommeraie}

La gestion d'une gommeraie consiste à faire parcourir un cycle évolutif complet au peuplement, depuis l'installation artificielle ou naturelle des jeunes pousses d'Acacias jusquà l'exploitation des vieux arbres, afin de tirer le maximum de profits de ses services, de ses produits et de ses sousproduits.

L'exploitation d'une gommeraie est réalisée selon une révolution de 20 à 23 ans comprenant les cycles suivants (Figure 3): 


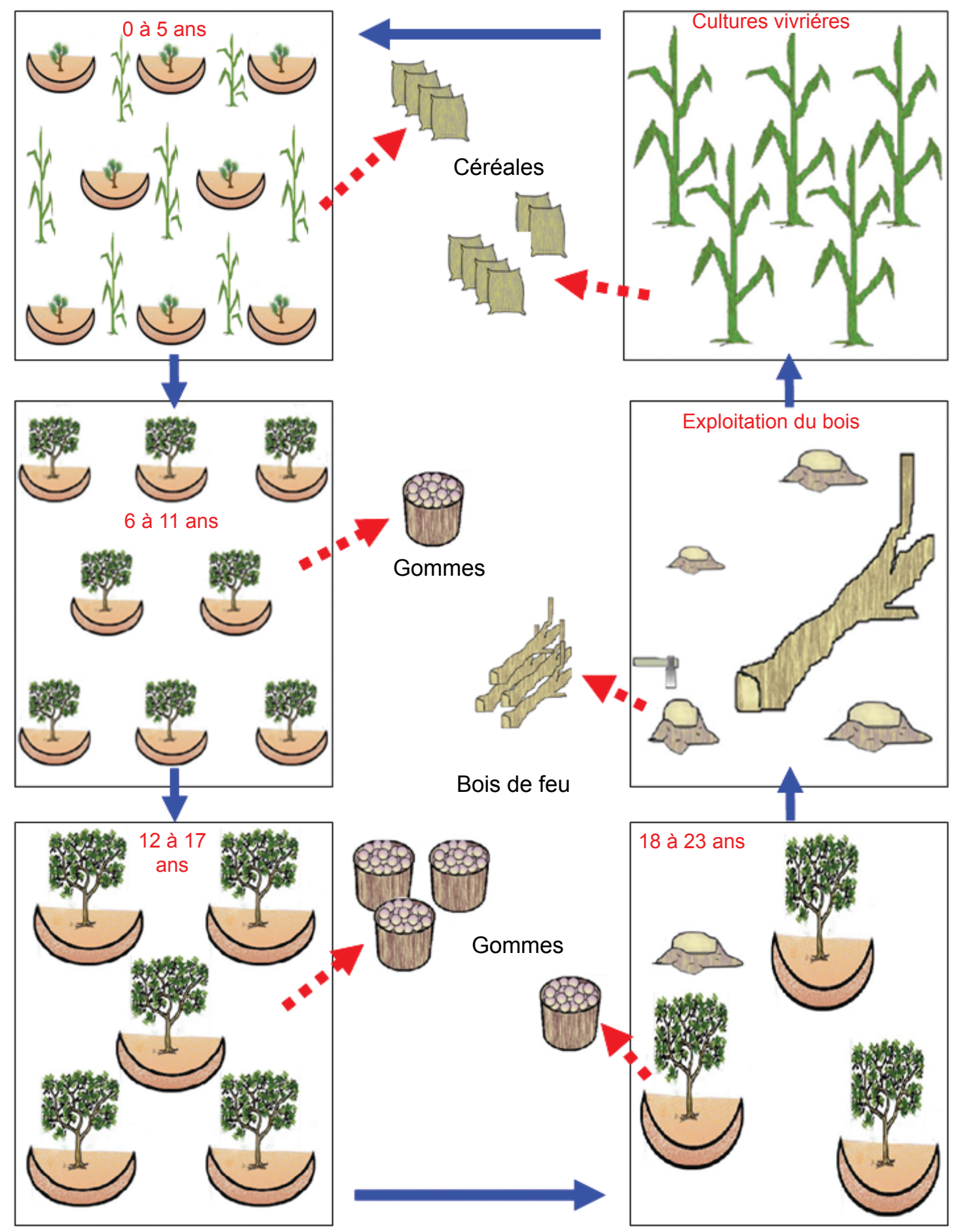

Figure 3. Cycle de gestion d'une gommeraie (d'après la FAO, 2004). 
Pendant 12 à 23 ans, la gommeraie fonctionne comme une jachère arborée. Entre 20 et 23 ans, les gommiers doivent être coupés (exploitation) et le sol de la gommeraie devient disponible pour quelques années de cultures. Les deux premières années de cultures sont très productives. Après la coupe de la gommeraie, on peut réintroduire les gommiers, soit par voie naturelle, soit par voie artificielle.

Tableau 2 : Cycle d'exploitation d'une gommeraie (Source : FAO, 2004).

\begin{tabular}{|c|c|}
\hline Cycle & Opérations \\
\hline 0 à 5 ans & $\begin{array}{l}\text { - Plantation jeune, issue de semis directs, de plants mis } \\
\text { en terre ou de rejets de souche : } \\
\text { - Entretien des plantules } \\
\text { - Pratique de cultures intercalaires } \\
\text { - } 44 \text { ans, la plante commence à produire la gomme } \\
\text { - A } 5 \text { ans, on peut appliquer la saignée }\end{array}$ \\
\hline 6 à 11 ans & $\begin{array}{l}\text { - Fin de la pratique des cultures intercalaires } \\
\text { - Activité principale production de la gomme } \\
\text { - Faible production de gomme }\end{array}$ \\
\hline 12 à 17 ans & $\begin{array}{l}\text { - Activité principale : production de gomme } \\
\text { - Période de production optimale de gomme }\end{array}$ \\
\hline 18 à 23 ans & $\begin{array}{l}\text { - Activité principale : production de gomme } \\
\text { - La production de gomme enregistre un déclin en } \\
\text { quantité et en qualité, avec des gommes de plus en } \\
\text { plus colorées. }\end{array}$ \\
\hline 20 à 23 ans & $\begin{array}{l}\text { - Exploitation de la gommeraie ; coupe à blanc étoc } \\
\text { (coupe rase) } \\
\text { - Production de bois de service, de bois d'énergie et de } \\
\text { branchages pour la confection des haies mortes de } \\
\text { protection. }\end{array}$ \\
\hline & - Début du nouveau cycle \\
\hline
\end{tabular}




\section{Module III \\ Techniques de production de la gomme}

\subsection{La gommose}

La gommose est le phénomène d'exsudation d'un liquide translucide à partir de l'écorce des acacias gommiers sous l'effet d'un traumatisme (blessure). L'exsudat, de nature pectique ou pecto-cellulosique, est appelé gomme. Il durcit au contact de l'air en prenant une couleur rose dorée. La gommose peut être naturelle ou provoquée.

- L'exsudation naturelle

Elle est une réponse à un stress subi par l'arbre. L'exsudation naturelle peut ne pas avoir lieu si le traumatisme naturel nest pas optimal.

- L'exsudation artificielle

La saignée consiste à pratiquer une blessure (appelée care) sur l’arbre en détachant un lambeau d'écorce pour provoquer artificiellement l'exsudation de la gomme. Il existe deux techniques de saignée : la méthode traditionnelle et la technique moderne.

\subsection{Les techniques de production}

\subsubsection{Matériel de saignée et de récolte}

- Instruments de nettoyage : coupe-coupe, cisailles, sécateurs.

- Instruments de protection : gants, lunettes.

- Instruments de saignée : saignette.

La saignette est constituée d'un manche en bois, surmonté d'une lame aux dimensions prédéfinies ( $8-10 \mathrm{~cm}$ de long sur 2-3 cm de large).

- Instrument de récolte

La gomme est collectée soit directement à la main lorsque les nodules sont situés à portée de main du collecteur soit à l'aide d'un instrument appelé " cueille-gomme » dans le cas contraire. Le cueille-gomme est 
composé d'un entonnoir muni d'une lame et d'un manche sur lequel l'ensemble du dispositif est fixé. Le manche traverse l'entonnoir par le milieu et déborde de quelques centimètres. Au cours de lopération de récolte, il faut éviter que les nodules tombent à terre.

- Matériel de conditionnement

La gomme récoltée est mise de préférence dans des sacs en toile propres.

- Autre matériel : bâche, natte.

Le matériel de saignée et de récolte peut être fabriqué localement. Des artisans ont été sélectionnés à Ouagadougou et à Kaya, qui confectionnent ce matériel suivant les normes préconisées. D’autres pourront être initiés en fonction des besoins et des zones de production afin de répondre à la demande des producteurs.

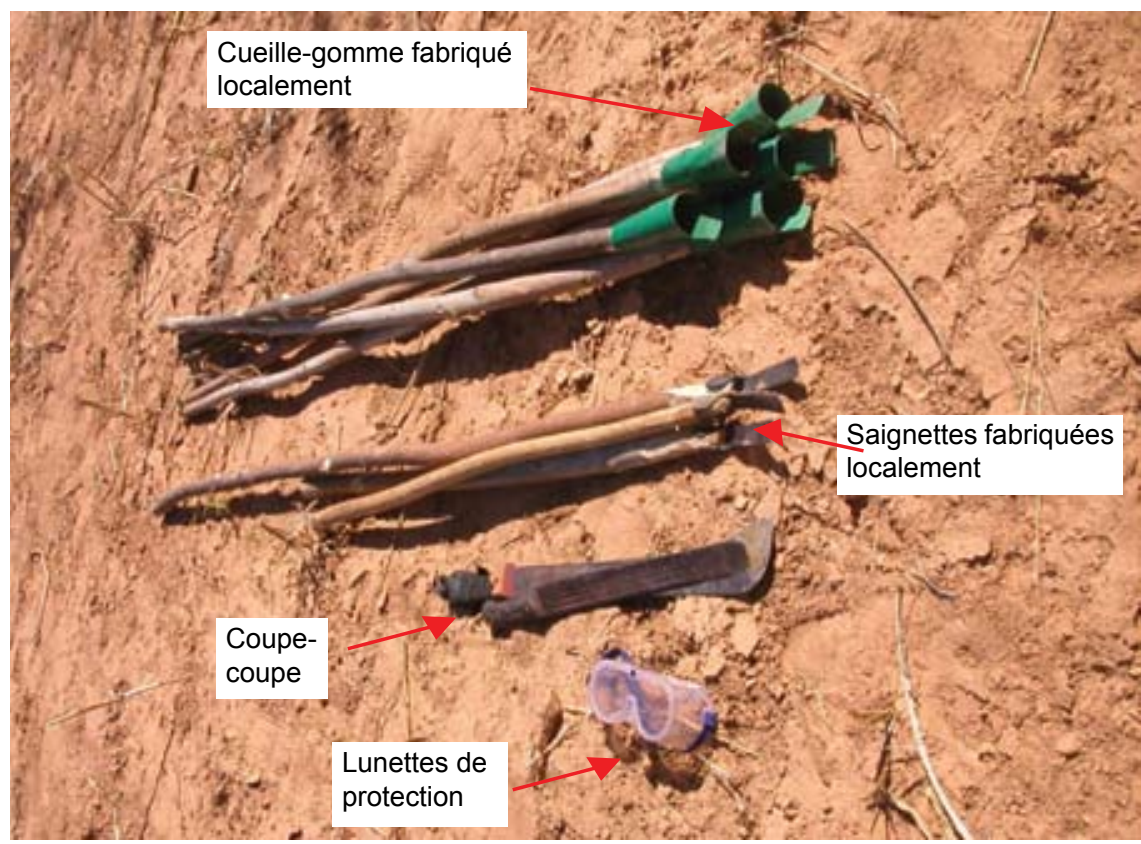

Photo 13. Outils couramment utilisés au Burkina Faso pour la saignée et la récolte de la gomme $(\odot \mathrm{DiFor})$. 


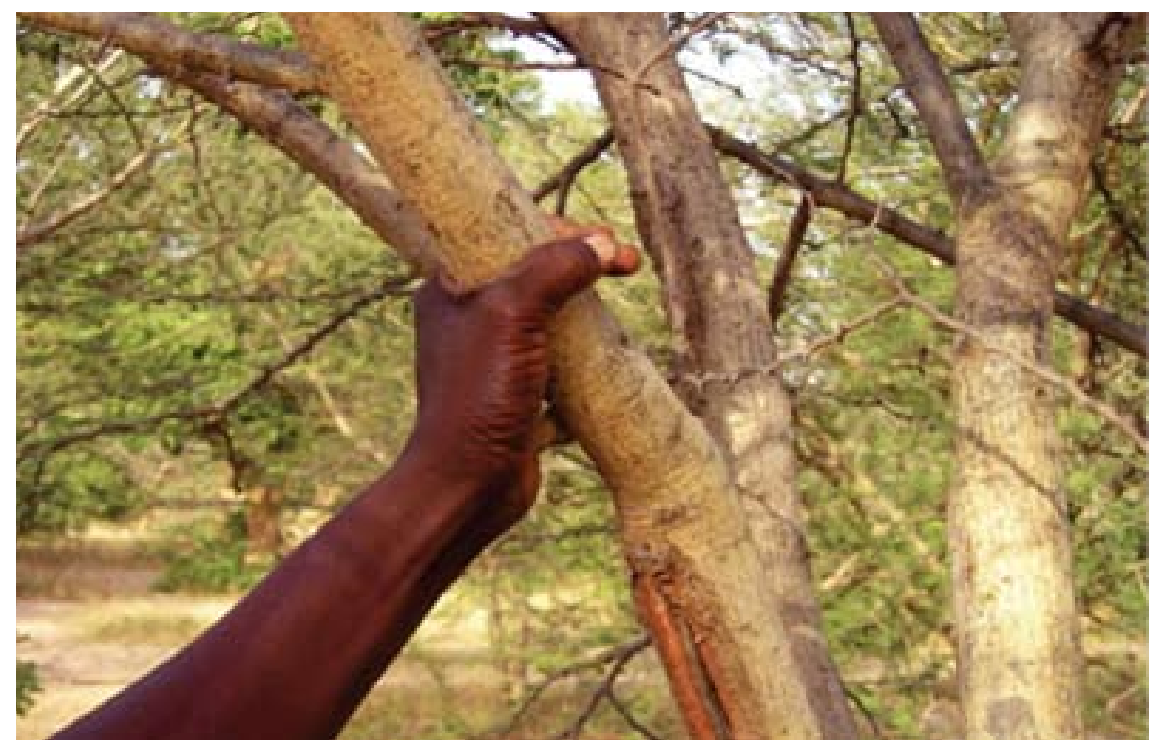

Photo 14. Indication de la grosseur requise pour une branche à saigner (C DiFor).

\subsubsection{La méthode traditionnelle de saignée}

Cette technique est dite abusive ou sauvage car effectuée sans aucune norme et est susceptible de provoquer la mort précoce de l'arbre.

\subsubsection{La technique moderne de saignée}

\section{Principes et normes}

Cette méthode est fondée sur des principes techniques et des normes à savoir la période de saignée, l'âge de l'arbre, le diamètre des branches entre autres.

- Dimensions des saignées

Le care (blessure issue de la saignée) mesure 15 à $30 \mathrm{~cm}$ de long et 2 à 3 $\mathrm{cm}$ de large.

- Grosseur des branches

La saignée est pratiquée sur des branches ayant un diamètre supérieur à $2,5 \mathrm{~cm}$, de préférence sur les diamètres de $4-5 \mathrm{~cm}$ (branches dont on ne peut faire le tour avec le pouce et l'index). 


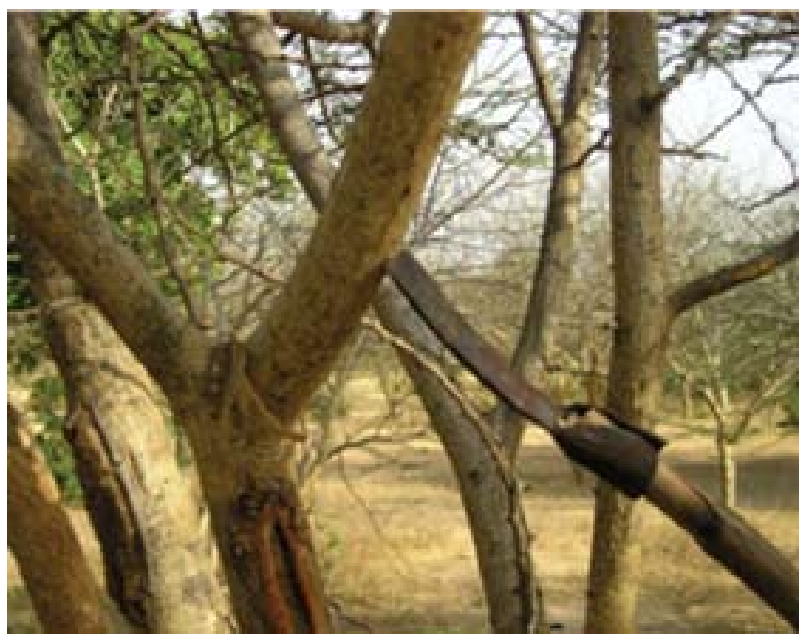

a) Enfoncer la lame de la saignette sous l'écorce de la branche.

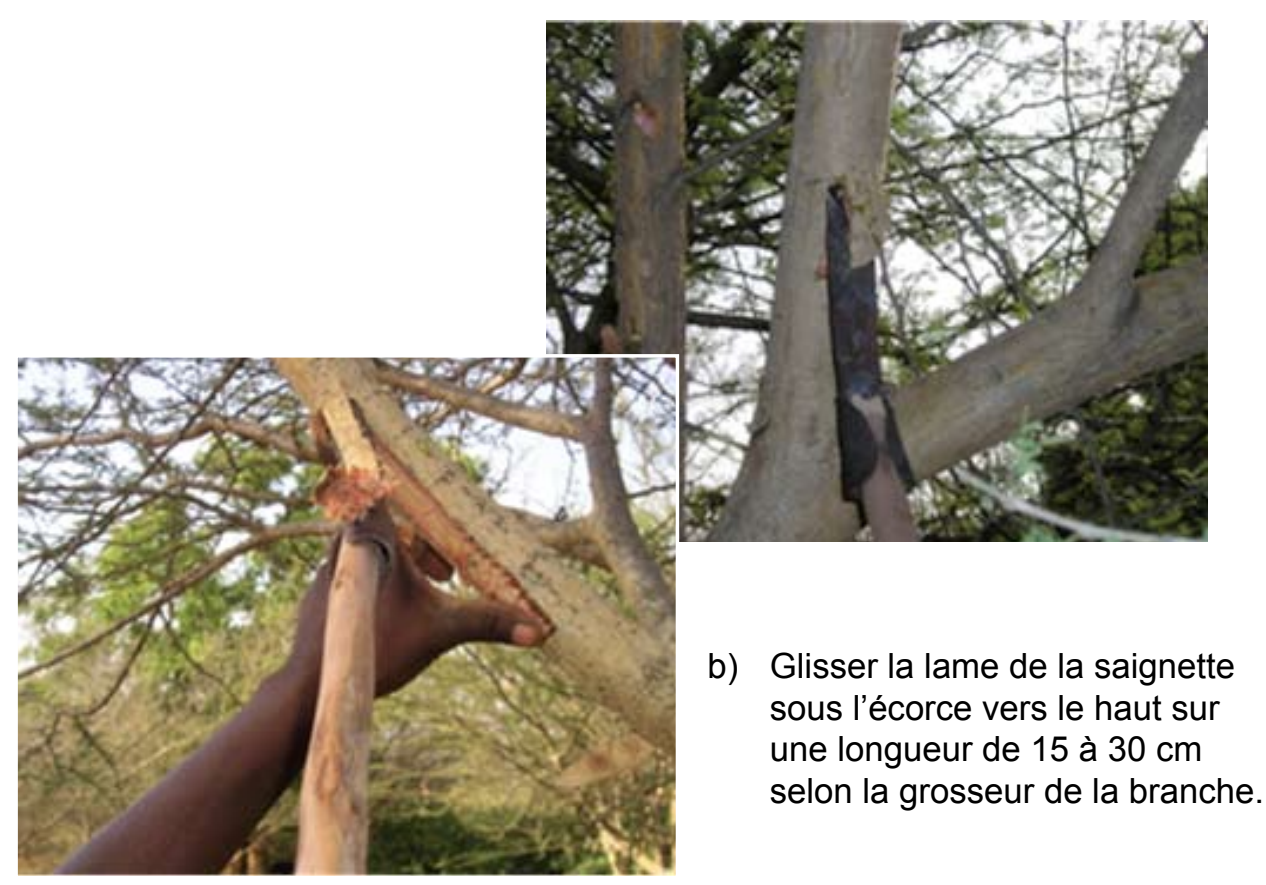




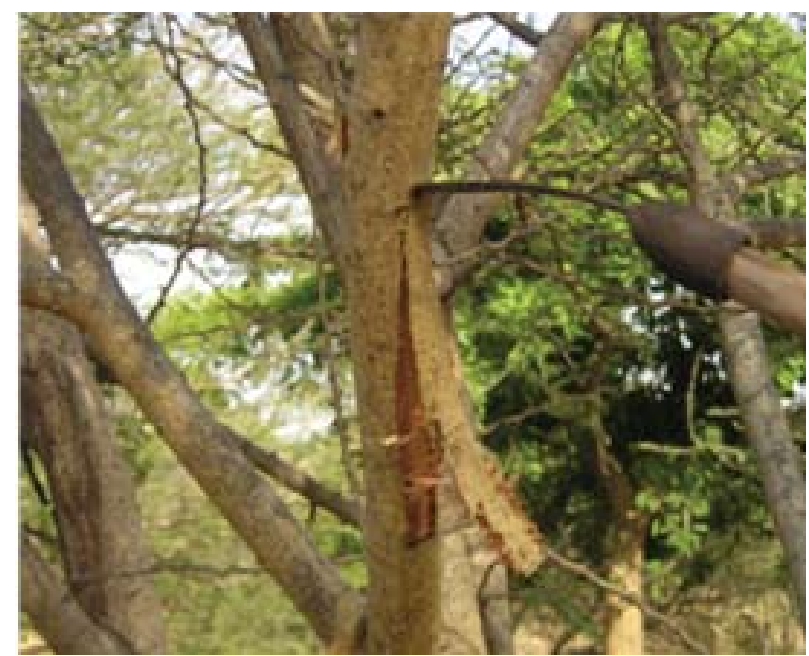

c) Couper la languette à la partie supérieure du care.

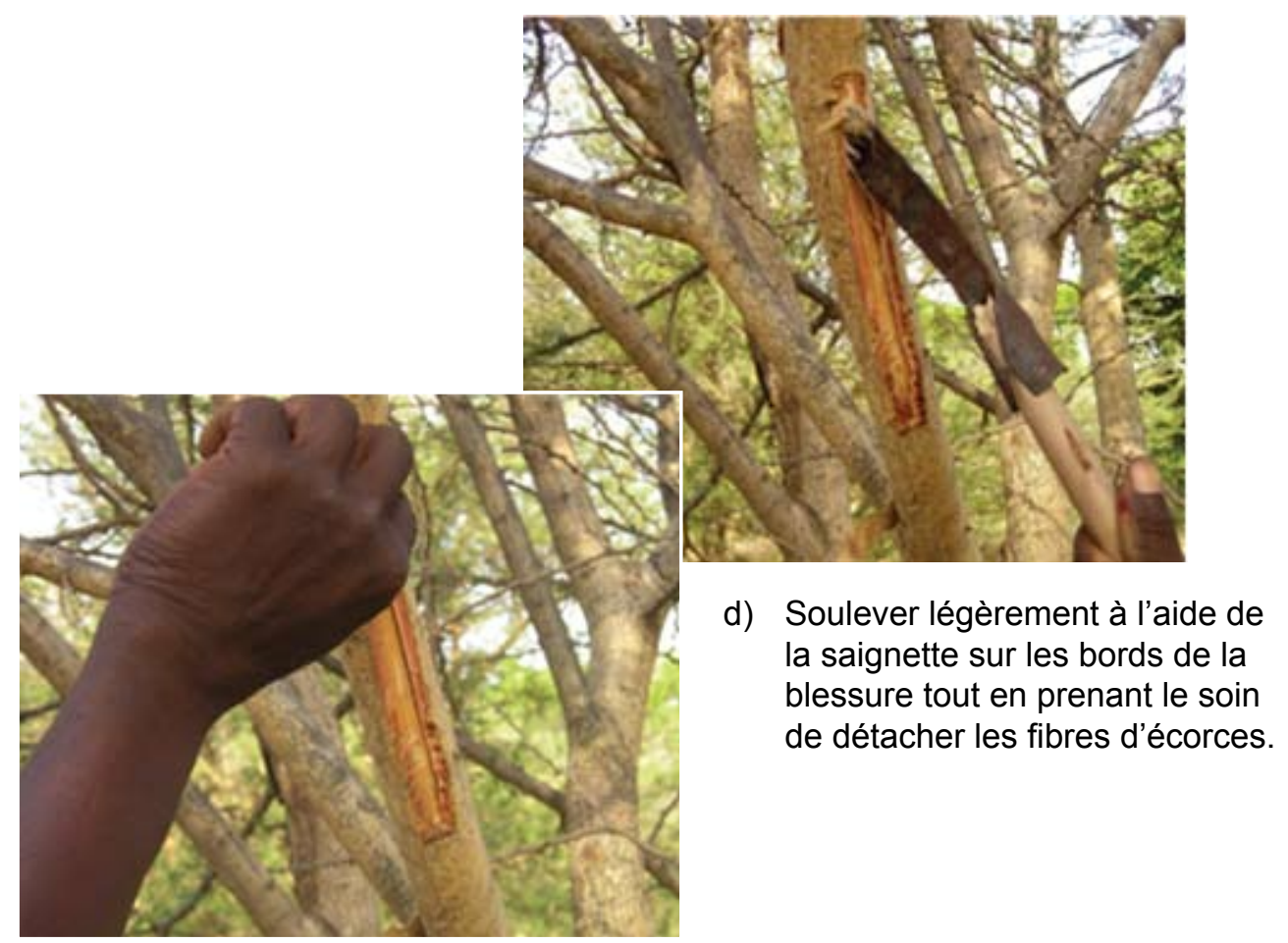

Photos 15 à 20. Les différentes étapes dans la réalisation de la saignée (@ DiFor). 
- Période de saignée

La période de saignée la plus propice se situe au début de la saison sèche, lorsque les arbres ont perdu au moins la moitié de leurs feuilles (octobre - novembre dans le cas du Burkina).

- Caractéristiques du gommier à saigner

Le gommier produit la gomme à partir de lâge de 3-4 ans. Age conseillé pour la saignée : arbre ayant au moins 5 ans.

- Nombre de saignées par branche

La saignée économique consiste à saigner sur toutes les branches de l'arbre ayant au moins $4 \mathrm{~cm}$ de diamètre tout en respectant le quotient de saignée.

La saignée rationnelle consiste à saigner $2 / 3$ du nombre total des branches de l'arbre, à raison de 1 à 2 cares par branche.

Il faut éviter une saignée anarchique qui entraînerait la mort de l’arbre. Le nombre total de saignées par arbre varie suivant le nombre de branche : 10 à 20 cares en moyenne par gommier.

\section{Ordre chronologique des opérations de saignée}

- Choisir les branches à saigner : éviter les branches dont on ne peut faire le tour de la circonférence avec le pouce et l'index;

- Tailler l'arbre afin de dégager les branches à saigner et de permettre au producteur d'y accéder facilement pour effectuer les différentes opérations ;

- Une fois la branche à saigner localisée, suivre les étapes de saignée suivantes ( $c f$. Photos 15 à 20) :

\subsection{Récolte de la gomme}

L'exsudation intervient quelques heures, voire plusieurs jours après la saignée. On distingue deux sortes de récolte de la gomme : la récolte d’amorce et la récolte proprement dite.

- La récolte d’amorce

Elle intervient une à deux semaines après la saignée (parfois 3 semaines ou plus en fonction de l'apparition de la gomme). 


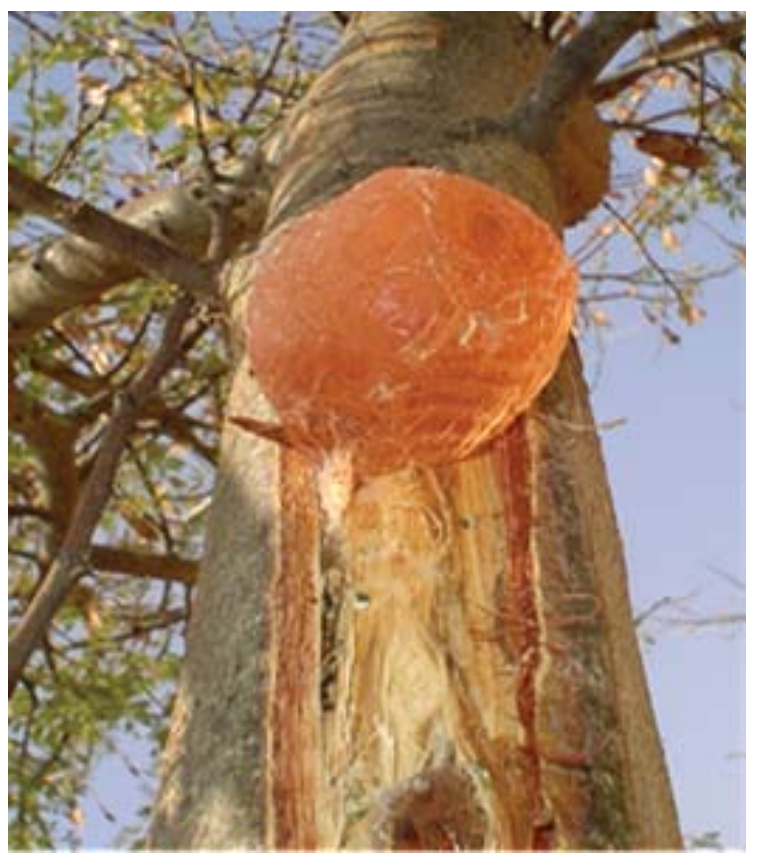

Photo 21. Care exsudant de la gomme (C) DiFor).

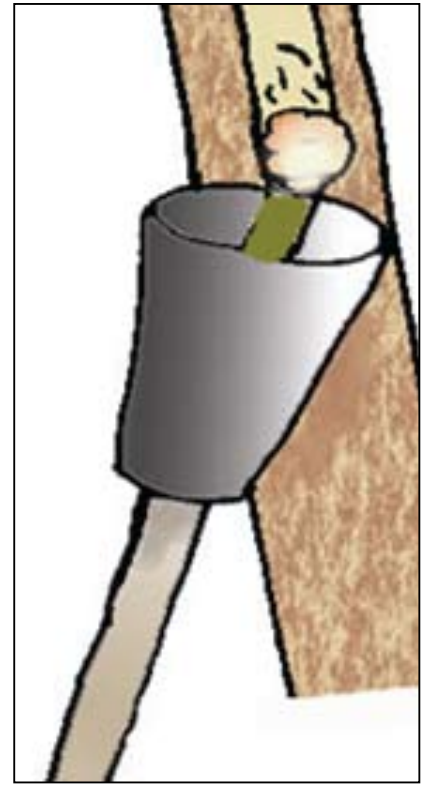

Figure 4. Opération de récolte de la gomme (Dessin : P. Bonkoungou).

- La récolte proprement dite

La récolte proprement dite est pratiquée toutes les deux ou trois semaines après la récolte d'amorce. Cet intervalle de temps permet le grossissement des boules de gomme qui est un critère déterminant de sa valeur marchande.

- La taille des nodules

La gomme est récoltée lorsqu’elle est suffisamment grosse, atteignant au moins la taille d'un jaune d'œuf (pour mieux répondre aux exigences du marché). Lorsqu'on enlève une boule de cette taille à la récolte d'amorce, aux récoltes suivantes, il se forme au même endroit des boules de taille supérieure.

- Durée de la récolte et nombre de récoltes

La récolte peut sétaler sur plusieurs mois, généralement de novembre à mars au Burkina Faso. On peut effectuer 3 à 7 récoltes dans la saison sur le gommier saigné. 


\subsection{Le traitement et le conditionnement de la gomme}

Le traitement est une opération très importante parce qu'il permet d'améliorer la qualité de la gomme et, partant, sa valeur marchande. Le traitement comprend les principales opérations suivantes : le nettoyage, le séchage, le tri et le conditionnement.

- Le nettoyage

La gomme fraîchement récoltée possède souvent des impuretés (débris d'écorce, de brindilles, morceaux de feuilles, etc.). Il faut donc nettoyer la gomme en la débarrassant de ces impuretés. Cette opération se fait aussitôt après la cueillette de la gomme (à l'état frais). Lopération devient difficile quand la gomme se dessèche.

- Le séchage

Le séchage permet de sécher le liquide qui se trouve encore à l'intérieur des nodules au moment de la récolte.

Après la récolte, la gomme est séchée à l'aire libre, à l'abri de la poussière et des impuretés pendant au moins une semaine.

- Le tri

Cette opération qui se fait manuellement, est complémentaire au nettoyage et vise non seulement à débarrasser la gomme de toute impureté mais aussi à opérer un tri des nodules suivant leur grosseur. On peut distinguer plusieurs classes de taille des nodules :

o les nodules supérieurs à $3 \mathrm{~cm}$ de diamètre ;

o les nodules de diamètre compris entre 1,5 et $3 \mathrm{~cm}$;

o les nodules de diamètre inférieur à $1,5 \mathrm{~cm}$, considérés comme concassés ;

o les brisures;

o la poudre de gomme.

Cette classification n'est pas une exigence des marchés.

- Le conditionnement

L'emballage pour conserver la gomme doit être propre et n'avoir jamais été utilisé à d'autres fins telles que le stockage de céréales ou autres. Il doit être perméable à l'air. Généralement la gomme est conditionnée dans des sacs en toile ou en fibre.

Il faut surtout éviter les sacs en plastique qui peuvent entraîner avec la durée de stockage, un changement de couleur de la gomme. Eviter aussi de stocker la gomme dans les mêmes endroits que des hydrocarbures, des insecticides et des engrais. 
La conservation de la gomme doit être aussi limitée que possible car une conservation prolongée favorise la perte en eau des nodules entraînant une diminution de leur volume. Si la conservation prolongée s’avère nécessaire, elle doit se faire dans un endroit sec et ombrageux loin des produits ou des matières qui peuvent dégager des odeurs.

Il est important d’étiqueter les sacs afin de faciliter leur identification par les acheteurs. Létiquetage porte le nom du produit, lespèce (nom botanique ou vernaculaire), la date et le lieu de la récolte, le nom du récolteur, le poids de la gomme.

Pour offrir des qualités compétitives, la gomme doit respecter les critères suivants :

- être propre : ne pas comporter de sable, de débris végétaux ni de feuilles ;

- être mûre : elle ne doit pas être récoltée trop rapidement après la saignée. Un temps de maturité de 15 jours est nécessaire si lon veut qu'elle ait toutes les qualités physico-chimiques recherchées ;

- être sèche : après la récolte, la gomme doit être séchée à l'air libre, à l'abri de la poussière et des impuretés pendant au moins une semaine ;

- être claire : autant que possible. Les nodules les plus clairs ont la cote et sont achetés en premier ;

- être pure : les gommes de différentes espèces d'acacia ne doivent pas être mélangées surtout quand il s'agit de gommes dures et de gommes friables.

C'est en respectant l'ensemble de ces qualités que le producteur peut exiger un prix élevé. La qualité génère une forte demande et un écoulement rapide.

Pour obtenir de la gomme de bonne qualité, il faut respecter les normes et techniques de production et n'utiliser que le matériel qui est adapté.

\subsection{Valorisation de la gomme}

La gomme est un produit de base très recherché. Elle est utilisée dans les industries pharmaceutiques et agroalimentaires, la cosmétique, le textile et l'industrie. 
La production de gomme est une activité susceptible de générer des revenus substantiels pour les agriculteurs qui la pratiquent. Elle a cependant besoin d'un bon accompagnement si lon veut être compétitif sur le plan mondial. Seule une gomme de bonne qualité présentée aux acheteurs retiendra lattention du marché.

Diversesconsidérations entrentenligne de compte pourlacommercialisation de la gomme.

\subsubsection{A l'échelle internationale}

Le marché international de la gomme est dominé par les pays de l'Afrique sub-sahariens. Le Soudan, premier producteur mondial, assure entre 60 et $70 \%$ de la production mondiale commercialisée. Il est suivi de près par le Tchad et le Nigeria. Viennent ensuite les pays émergents comme le Sénégal, le Mali, le Niger, la Mauritanie et de potentiels pays émergeants comme le Burkina Faso.

Des études menées par Macrae et Merlin (2002) ont montré que la consommation de la gomme a augmenté, passant de 40000 à 50000 tonnes de 1995 à 2000. Les mêmes sources indiquent une progression du marché avec des prévisions d'accroissement de $5 \%$ et un seuil de production de 90000 tonnes en 2010. Cette perspective renforce les opportunités commerciales pour les pays déjà exportateurs et en ouvre pour ceux comme le Burkina qui ont besoin de conditions plus favorables pour développer la filière.

La promotion de la gomme arabique se fait au niveau mondial par Network for Natural Gums and Resins in Africa (NGARA) qui est un réseau regroupant 15 pays : le Burkina Faso, le Cameroun, l'Erythrée, l'Ethiopie, le Kenya, le Mali, la Mauritanie, le Niger, le Nigeria, l'Ouganda, le Sénégal, le Soudan, la Somalie, la Tanzanie et le Tchad. Le NGARA poursuit les objectifs suivants :

- promouvoir les échanges d'informations sur la production, la manutention, le marketing de la gomme arabique entre les producteurs et les partenaires ;

- faciliter l'accès à la technologie et à l'information ;

- soutenir la recherche dans les domaines prioritaires pour le développement de la filière ;

- promouvoir des liens entre les producteurs, les transformateurs et les utilisateurs de la gomme arabique. 


\subsubsection{Le marché de la gomme au Burkina Faso}

\section{Les acteurs de la filière}

En 1995, le gouvernement a adopté une politique de foresterie basée sur la valorisation des ressources forestières en vue de contribuer à l'amélioration des conditions de vie des producteurs. Cette politique a favorisé lorganisation des acteurs de la production et de la commercialisation de la gomme. Dans la foulée de cette initiative, l'Union Européenne a soutenu en 1996 le lancement de la campagne de promotion de la gomme arabique qui avait pour but de renforcer les capacités techniques des cadres forestiers et des producteurs d'une part et d'évaluer le potentiel physique des acacias gommiers dans le pays d'autre part.

Deux types d'acteurs animent la filière gomme au Burkina Faso.

Le premier groupe est constitué par les acteurs qui prennent part directement au fonctionnement de la filière. On y retrouve les producteurs, les collecteurs, les commerçants et les exportateurs.

Les partenaires techniques (structures de l'Etat, ONG, Projets de développement, etc.) constituent le second groupe.

\section{Les circuits de production et de commercialisation}

Les producteurs produisent la gomme avec lappui des partenaires au développement et des structures étatiques. La gomme produite est achetée par des groupements, associations et commerçants locaux puis revendue à des intermédiaires qui peuvent être des exportateurs nationaux ou des commerçants étrangers. Les circuits de commercialisation, au-delà de ce stade, se poursuivent hors du Burkina et se terminent dans les industries de transformation qui élaborent différents produits finis (ou semi finis).

\section{- Les exportateurs}

Les exportateurs actuellement en activité au Burkina sont l'APEGA (Association Professionnelle des Exportateurs de Gomme Arabique) et Gomburki SARL. Ils achètent la gomme collectée dans les zones de production et l'acheminent à Ouagadougou pour l'exportation vers l'Europe ou d'autres destinations. Ces deux acteurs disposent chacun d'un réseau d'intermédiaires chargés d'acheter la gomme dans des sites bien définis, de la stocker dans un point central et de l'acheminer vers Ouagadougou. Des avances de payement sont parfois octroyées aux producteurs pour inciter la collecte. 


\section{- Les commerçants et acheteurs}

Ce sont souvent des négociants qui achètent la gomme auprès des producteurs. Outre les nationaux, on retrouve aussi des négociants nigériens qui opèrent dans les provinces du Yagha et de la Komandjari. On note également des arrivées d'acheteurs en provenance du Mali et du Nigeria. Certains acheteurs exigent la pureté tandis que d'autres achètent de la gomme tout venant (cas des négociants nigériens). Ces commerçants pratiquent également différents prix à l'achat (de 300 à 800 F CFA l’assiettée).

\subsubsection{Les applications de la gomme au Burkina Faso}

A l'instar des autres pays Africains, la gomme a plusieurs utilisations dans la vie quotidienne au Burkina Faso.

Elle est vendue sur le marché par des commerçants locaux avec des instruments de mesures traditionnels sous plusieurs formes.

Elle intervient dans la constitution de matières servant à la médecine traditionnelle humaine et animale sous forme de pansement gastrique.

La gomme sert également à préparer de l'encre et des colorants pour la teinture, la décoration des objets d'art et donc occupe une grande place dans l'artisanat utilitaire.

Elle intervient aussi dans la préparation de certains plats traditionnels très prisés. Elle sert à lempesage des tissus. Cependant, la quantité de gomme utilisée pour ces différents usages est très négligeable et ne nécessite pas une exploitation à grande échelle et un développement de la filière. 


\section{Documents consultés}

Balima R., 2000. Essai de production de gomme arabique dans les peuplements naturels de Acacia spp. Rapport technique $\mathrm{n}^{\circ} 35$, CNSF. $43 \mathrm{p}$.

Bognounou O., 2000. Ethnobotanique. Protocole daccord technique entre I'INERA et Monsieur Bognounou Ouétian. 10 p.

Direction de la Foresterie Villageoise et de l'Aménagement Forestier, 1999. Guide de formation à l'usage des agents Acacia senegal et production de gomme arabique.

Direction des Forêts, 2006. Plan décennal de développement de la filière gomme arabique au Burkina Faso.

Fagg C.W., Allison G.E., 2004. Acacia senegal and the gum arabic trade. Oxford Forestry Institute. Tropical Forestry Papers $42: 1-39$.

FAO, 1999. Etude d'impact du travail des charrues « Delfino » et " treno » sur la récupération des terres fortement dégradées.

FAO, 2004. Gommiers et gomme arabique, manuel de terrain.

FAO, 2005. Programme de formation régional.

Kaboré A., 1998. Etude socio-économique de la gestion de l'Acacia senegal (L.) Willd. au Burkina Faso. Mémoire de fin détudes présenté en vue de l'obtention du diplôme d'ingénieur du développement rural, option Eaux et Forêts. IDR/UPB, CNSF, 96 p.

Macrae J., Merlin G., 2002. The prospects and the constraints of Development of Gum Arabic in Sub-Saharan Africa. A document based on the Available literature and field trips to Chad, Mali and Niger. Wold Bank, $47 \mathrm{p}$.

Maydell H.-J. von, 1990. Arbres et arbustes du Sahel leurs caractéristiques et leurs utilisations. Weikersheim, Margraf, $531 \mathrm{p}$.

Ministère de l'Environnement et de l'Eau, 2001. Manuel de foresterie villageoise.

Ministère de l'Environnement et de l'Eau, 2002. Manuel de pépinière villageoise. 
Nacoulma O. 1996. Plantes médicinales et pratiques médicalestraditionnelles au Burkina Faso cas du plateau central. Thèse de doctorat d'Etat, Tome II, Université de Ougadougou, Burkina Faso.

Nikiéma A., Poda D. et Ouédraogo M., 1997. Inventaire et cartographie de peuplements naturels de Acacia senegal (L.) Willd. Au Burkina Faso. Rapport technique n²3. ISSN 1018 - 7065, CNSF et Projet 7 ACP BKF/031. $29 \mathrm{p}$.

Projet Opération Acacia, 2005. Manuel de lacteur de la filière gomme arabique la qualité de la gomme.

Stone R., 1998. Quel est votre rôle ? Guide à l'intention des responsables de la formation à la gestion des aires protégées. Série Biodiversité africaine, $\mathrm{N}^{\circ} 5$ Washington, D.C. Biodiversity Support Production.

Weigel J., 1994. Agroforesterie pratique à l'usage des agents de terrain en Afrique tropicale sèche. 


a gomme est un produit aussi vieux que le monde. Dans l'Egypte ancienne, elle était utilisée pour la fabrication de l'encre, des hiéroglyphes, dans la poterie. De nos jours, ses propriétés physico-chimiques compatibles avec de multiples utilisations dans les industries agroalimentaires, pharmaceutiques, cosmétiques et autres en font un produit de grand commerce international. Bien que la demande mondiale de gomme ait enregistré des fluctuations importantes au cours du 20 siècle, avec des extrêmes à 60 000 tonnes à la fin des années 60 et environ 20000 tonnes au début des années 90, les tendances sont maintenant à la hausse et les seules exportations des pays africains dépassaient les 40000 tonnes vers la fin des années 2000 (Fagg et Allison, 2004).

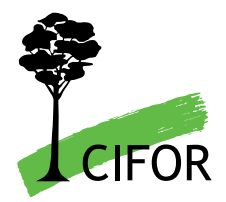

Esdi

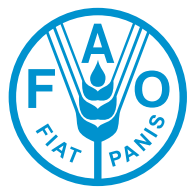

